\title{
CATEGORICAL METHODS IN GRADED RING THEORY
}

\author{
ANGEL DEL RÍO* \\ A la memoria de Pere Menal
}

\begin{abstract}
Let $G$ be a group, $R$ a $G$-graded ring and $X$ a right $G$-set. We study functors between categories of modules graded by $G$-sets, continuing the work of $[M]$. As an application we obtain generalizations of Cohen-Montgomery Duality Theorems by categorical methods. Then we study when some functors introduced in [M] (which generalize sorne functors ocurring in [D1], [D2] and [NRV]) are separable. Finally we obtain an application to the study of the weak dimension of a group graded ring.
\end{abstract}

\section{Introduction and Notation}

The study of group-graded rings has been the ground for the research of many authors last years. Different methods have been elaborated to investigate propertics of these rings. One of the most successful tools is that given by Cohen-Montgomery Duality Theorems [CM]. Furthermore, some categorical methods have been introduced by other authors which have been very useful. For instance the study of separable functors, introduced in [NVV], has caused nice theorems in the field.

Quite often the methods invented have been introduced in the particular case when the grading group is finite. This is the case of the two methods mentioned above. Then some efforts have produced different approaches to the general case. See $[\mathbf{Q}],[\mathrm{B} 1],[\mathrm{B2}],[\mathbf{A N}]$ and [NRV] for some extensions of Cohen-Montgomery Duality Theorem and [Ra] for separable functors.

In [NRV] the authors introduce a category $g r-(R, X, G)$ associated to a $G$-graded ring $R$ ( $G$ being a group) and a right $G$-set $X$. The most

*Partially supported by CICYT PB90-0300. 
important example appears when $X$ is the set $G / H$ of right $H$-coscts. This category appears to be useful in the study of the ring $R$.

Let $G$ and $G^{\prime}$ be two groups, $R$ a $G$-graded ring, $R^{\prime}$ a $G^{\prime}$-graded ring, $X$ a right $G$-set and $X^{\prime}$ a right $G^{\prime}$-set. In [M] covariant adjoint functors between the categories $g r-(R, X, G)$ and $g r-\left(R^{\prime}, X^{\prime}, G^{\prime}\right)$ are studied, generalizing some results of [Rí2].

Let $\rho: R \rightarrow R^{\prime}$ be a ring homomorphism, $\xi: X \rightarrow X^{\prime}$ a map and $\gamma: G \rightarrow G^{\prime}$ a group homomorphism and assume that for every $g \in G$ and $x \in X, \rho\left(R_{g}\right) \subseteq R_{\gamma(g)}$ and $\xi(x) \gamma(g)=\xi(x g)$. Associated to $T=(\rho, \xi, \gamma)$ there is a pair of adjoint functors $T^{*}=g r-(R, X, G) \rightarrow g r-\left(R^{\prime}, X^{\prime}, G^{\prime}\right)$ and $T_{*}: g r-\left(R^{\prime}, X^{\prime}, G^{\prime}\right) \rightarrow g r-(R, X, G)$ for which many functors occurring in Graded Clifford Theory (cf. [D1], [D2], [GN], [NV2]) as well as induced and coinduced functors (cf. $[\mathrm{MN}])$ are particular cases.

After some definitions and notations we recall in Section 1 results of $[\mathrm{M}]$ on covariant adjoint functors and point out some similar fact on contravariant adjoint functors which extend some results of [MR]. We finish Section 1 with some applications to the functors $T^{*}$ and $T$. associated to a context $T=(\rho, \xi, \gamma)$ as in the previous paragraph.

The results of Section 1 set up the framework to give a description of all the equivalences and Morita dualities (in the sense of Colby-Fuller [CF]) between categories of the type $g r-(R, X, G)$ using similar methods as in [Ri2] and [MR], This is done without proofs in Section 2.

In Section 3 we apply the tools of Section 1 to extend CoheriMontgomery Duality Theorems to our setting by using categorical methods. Namely, we show how the category $g r-(R, X, G)$ can be seen as the full subcategory of a category of right modules generated by an ideal. Then we prove new versions of Cohen-Montgomery Duality Theorems for actions and coactions. All the previous versions of thesc Duality Theorems can be obtained as particular cases. Furthermore, some equivalences of categories are given which will be useful in applications.

Section 4 is devoted to study when $T^{*}$ and $T_{*}$ are separable. We give some arithmetical tests for the matter. When $R=R^{\prime}$ and $\rho$ is the identity map on $R, T^{*}$ and $T_{*}$ are isomorphic to the functors $T_{\xi}$ and $S^{\xi}$ introduced in [NRV] and, in this case, $T_{\xi}$ is always separable while the test of separability for $S^{\xi}$ can be simplified.

We finish the paper with an application to the weak dimension of a group graded ring.

The author would like to express his gratitude to Claudia Menini for her very intcresting suggestions.

All rings are supposed to be associative with unit and all modules are 
unital.

For a ring $R, \bmod -R$ will denote the category of right $R$-modules and $R$-mod the category of left $R$-modules.

The notation $M_{R}$ (resp. ${ }_{R} M$ ) will be used to emphasize that $M$ is a right (resp. left) $R$-module.

In the sequel $G$ will stand for a multiplicative group and $R=\underset{g \in G}{\oplus} R_{g}$ for a $G$-graded ring. The unit of $G$ will be denoted by $e$ while 1 will denote the unit of $R$.

Let $X$ be a right (resp. left) $G$-set. For any $g \in G$ and any $x \in X, x g$ (resp. $g x$ ) denotes the action of $g$ on $x$.

Following the notation introduced in [NRV], $g r-(R, X, G)$ will denote the category of $X$-graded right $R$-module. That is, the objects of $g r-(R, X, G)$ are right $R$-modules $M$ with a decomposition into additive subgroups $M=\underset{x \in X}{\oplus} M_{x}$ such that for every $g \in G$ and every $x \in X, M_{x} R_{g} \subseteq M_{x g}$. Given $M, N$ objects in $g r-(R, X, G)$, the set of morphisms from $M$ to $N$ in $g r-(R, X, G)$ is

$$
\begin{aligned}
\operatorname{Hom}_{g r-(R, X, G)}(M, N)= \\
=\left\{f \in \operatorname{Hom}_{R}(M, N) \mid f\left(M_{x}\right) \subseteq N_{x} \text { for every } x \in X\right\} .
\end{aligned}
$$

Similarly, if $X$ is a left $G$-set then $(G, X, R)-g r$ will denote the category of $X$-graded left $R$-modules. If $M \in(G, X, R)-g r$ and $x \in X$, then the $x-$ th component of $M$ will be denote by ${ }_{x} M$.

If $G$ is considered as a right $G$-set by regular action, then $g r-(R, G, G)$ is just $g r-R$. If $X$ is a singleton, then $g r-(R, X, G)$ is $\bmod -R$.

Let $H$ be a subgroup of $G$ and consider $G$ acting on $G / H=\{H a \mid a \in$ $G\}$ by $(H a) g=H a g$, then $g r-(R, G / H, G)$ is denoted by $g r-(R, G / H)$.

Given $M \in g r-(R, X, G)$ (resp. $M \in(G, X, R)-g r), m \in M$ and $x \in X, m_{x}$ (resp. ${ }_{x} m$ ) will denote the $x-t h$ homogeneous component of $m$. That is, $m_{x}$ is defined by $m=\sum_{x \in X} m_{x}$ with $m_{x} \in M_{x}$ for every $x \in X$. If $F$ is a subset of $X$, then $M_{F}=\underset{x \in F}{\oplus} M_{x}$ and $m_{F}=\sum_{x \in F} m_{x}$.

The support of an $X$-graded right $R$-module is defined to be the set $\operatorname{Supp}(M)=\left\{x \in X \mid M_{x} \neq 0\right\}$. Also the support of $m \in M$ is the set $\operatorname{Supp}(m)=\left\{x \in X \mid m_{x} \neq 0\right\}$.

When we refer to the support or the homogeneous component of degree $g \in G$ of $R$ (or of an element of $R$ ) we will consider $R$ as a $G$-graded right (or left) $R$-module.

If $X$ is a right $G$-set then $X$ has a canonical structure of left $G$-set as follows: For any $g \in G ; x \in X, g x=x g^{-1}$. We will refer to both right 
and left action without specific mention on the side when it is clear from the context.

Given $M \in g r-R$ and $x \in X$, the $x-t h$ suspension of $M$ is defined as the $X$-graded right $R$-module $M(x)$ which is equal to $M$ as right $R$-modulc with the grading $M(x)_{y}=\underset{x g=y}{\oplus} M_{g}$. If $M \in R-g r, X$ is a left $G$-set and $x \in X$, then the $x-t h$ suspension of $M$ is the $X$-graded left $R$-module $(x) M$ which is equal to $M$ as a left $R$-module with the $X$-grading ${ }_{y}(x) M=\underset{g x=y}{\oplus} M_{g}$.

\section{Adjoint functors}

Throughout this section $G$ and $G^{\prime}$ will be two groups, $X$ a right $G$-set and $X^{\prime}$ a right $G^{\prime}$-set. Moreover $R$ will be a $G$-graded ring and $R^{\prime}$ a $G^{\prime}$-graded ring.

In this first section we will give an explicit description of the covariant functors between $g r-(R, X, G)$ and $g r-\left(R^{\prime}, X^{\prime}, G^{\prime}\right)$ and the contravariant functors between $(G, X, R)-g r$ and $g r-\left(R^{\prime}, X^{\prime}, G^{\prime}\right)$. This description has been obtained in $[\mathbf{R} \mathbf{i} 2]$, for the covariant case, when $X=G$ and $X^{\prime}=G^{\prime}$ with regular action, and generalized for arbitrary $G$-sets in $[\mathrm{M}]$. For the contravariant case, when $X=G$ and $X^{\prime}=G^{\prime}$, see [MR].

In the last part of the section we consider some instances of adjoint functors introduced in $[\mathbf{M}]$.

Definition. Given $M \in g r-(R, X, G)$ and $N \in(G, X, R)-g r$, $M \hat{\otimes}_{R} N$ will denote the additive subgroup of $M \otimes_{R} N$ generated by the elements of the form $m \otimes n$ where $x \in X, m \in M_{x}$ and $n \in{ }_{x} N$.

Lemma 1.1. (a) $R(x) \hat{\otimes}_{R} N \cong{ }_{x} N$ for every $N \in(G, X, R)-g r$. Moreover this isomorphism is natural in both variables.

(b) Let $\left\{M_{i} \mid i \in I\right\}$ be a family of objects in $g r-(R, X, G)$ and $N \in$ $(G, X, R)-g r$, then $\left(\underset{i \in I}{\oplus} M_{i}\right) \hat{\otimes}_{R} N \cong \underset{i \in I}{\oplus}\left(M_{i} \hat{\otimes}_{R} N\right)$.

Proof: (a) Let $\Phi: R(x) \otimes_{R} N \rightarrow N$ be the canonical isomorphism. If $r \in R_{g} \cap R(x)_{y}$ and $n \in{ }_{y} N$ then $x g=y$ and hence $x=g y$. Therefore $\Phi(r \otimes n)=r n \in{ }_{x} N$. Thus $\Phi\left(R(x) \hat{\ominus}_{R} N\right) \subseteq{ }_{x} N$. On the other hand, if $n \in{ }_{x} N$, then $n=\Phi(1 \otimes n)$ and $1 \otimes n \in R(x) \hat{\otimes}_{R} N$ because $1 \in R(x)_{x}$.

(b) Straightforward.

Definition. Let $Z$ be a $G$ - $G^{\prime}$-set. A $Z$-graded $R$ - $R^{\prime}$-bimodule is an $R$ - $R^{\prime}$-bimodule $M$ with a $Z$-grading, $M=\underset{z \in Z}{\oplus} M_{z}$ such that $R_{g} M_{z} R_{g^{\prime}}^{\prime} \subseteq$ 
$M_{g z g^{\prime}}$ for every $g \in G, z \in Z$ and $g^{\prime} \in G^{\prime}$.

The set $X \times X^{\prime}$ has a canonical structure of $G$-G'-set given by:

$$
g\left(x, x^{\prime}\right) g^{\prime}=\left(g x, x^{\prime} g^{\prime}\right) \quad\left(g \in G, x \in X, x^{\prime} \in X^{\prime}, g^{\prime} \in G^{\prime}\right) .
$$

Let $P=\underset{\left(x, x^{\prime}\right) \in X \times X^{\prime}}{\oplus} P_{\left(x, x^{\prime}\right)}$ be an $X \times X^{\prime}$-graded $R$ - $R^{\prime}$-bimodule.

For every $x \in X,{ }_{x} P=\underset{x^{\prime} \in X^{\prime}}{\oplus} P_{\left(x, x^{\prime}\right)}$ is a submodule of $P_{R^{\prime}}$ and $\left.{ }_{{ }_{x}} P\right)_{x^{\prime}}=P_{\left(x, x^{\prime}\right)}\left(x^{\prime} \in X^{\prime}\right)$ defines an $X^{\prime}$-grading in ${ }_{x} P$ such that ${ }_{x} P \in g^{r}-\left(R^{\prime}, X^{\prime}, G^{\prime}\right)$. Moreover $P$ is the coproduct of the family $\left\{{ }_{x} P \mid x \in X\right\}$ in $g^{r}-\left(R^{\prime}, X^{\prime}, G^{\prime}\right)$.

Similarly for every $x^{\prime} \in X^{\prime}, P_{x^{\prime}}=\underset{x \in X}{\oplus} P_{\left(x, x^{\prime}\right)} \in(G, X, R)-g r$ and $P$ is the coproduct of $\left\{P_{x^{\prime}} \mid x^{\prime} \in X^{\prime}\right\}$ in $(G, X, R)-g r$.

We are going to define three functors associated to the $X \times X^{\prime}$-graded $R$ - $R^{\prime}$-bimodule $P$.

The functor $-\hat{\otimes}_{R} P: g r-(R, X, G) \rightarrow g r-\left(R^{\prime}, X^{\prime}, G^{\prime}\right)$.

It associates to $M \in g r-(R, X, G)$ the right $R^{\prime}$-module $\left(-\hat{\otimes}_{R} P\right)(M)=$ $M \hat{\otimes}_{R} P$, considered as a submodule of $\left(M \oplus_{R} P\right)_{R}$, with the $X^{\prime}$-grading $\left.M \hat{\otimes}_{R} P\right)_{x^{\prime}}=M \hat{\otimes}_{R} P_{x^{\prime}}\left(x^{\prime} \in X^{\prime}\right)$.

The functor $-\hat{\otimes}_{R} P$ associates to $f \in \operatorname{Hom}_{g^{r}-(R, X, G)}(M, N)$ the map $f \hat{\otimes}_{R} P: m \otimes p \mapsto f(m) \otimes p$.

The functor $H\left(P_{R^{\prime}},-\right): g r-\left(R^{\prime}, X^{\prime}, G^{\prime}\right) \rightarrow g r-(R, X, G)$.

It associates to $M \in g r-\left(R^{\prime}, X^{\prime}, G^{\prime}\right)$ the right $R$-module

$$
\begin{aligned}
& H\left(P_{R^{\prime}}, M\right)= \\
& \quad=\left\{f \in \operatorname{Hom}_{g^{r}-\left\langle R^{\prime}, X^{\prime}, G\right)}(P, M) \mid f\left({ }_{x} P\right)=0 \text { for almost all } x \in X\right\}
\end{aligned}
$$

considered as a submodule of $\operatorname{Hom}_{R^{t}}(P, M)_{R}$ with the $X$-grading:

$$
\begin{aligned}
& H\left(P_{R^{\prime}}, M\right)_{x}= \\
= & \left\{f \in \operatorname{Hom}_{g r-\left(R^{\prime}, X^{\prime}, G^{\prime}\right)}(P, M) \mid f\left({ }_{y} P\right)=0 \text { for all } y \in X-\{x\}\right\}(x \in X) .
\end{aligned}
$$

The functor $H\left(P_{R},-\right)$ associates to $f \in \operatorname{Hom}_{g r-\left\langle R^{\prime}, X^{\prime}, G^{\prime}\right)}\left(M^{\prime}, N^{\prime}\right)$, the map $H\left(P_{R^{\prime}}, f\right): H\left(P_{R^{\prime}}, M\right) \rightarrow H\left(P_{R^{\prime}}, N\right)$ given by: $H\left(P_{R^{\prime}}, f\right)(\varphi)=$ $f \circ \varphi$ for all $\varphi \in H\left(P_{R^{\prime}}, M\right)$. 
The functor $H\left(-, R_{R} P\right):(G, X, R)-g r \rightarrow g r-\left(R^{\prime}, X^{\prime}, G^{\prime}\right)$.

It associates to $M \in(G, X, R)-g r$, the right $R^{\prime}$-module

$H\left(M,{ }_{R} P\right)=$

$=\left\{f \in \operatorname{Hom}_{G, X, R)-g r}(M, P) \mid f(m) \subseteq P_{E}\right.$ for some finite subset $\left.F \subseteq X^{\prime}\right\}$

considered as a submodule of $\operatorname{Hom}_{R}(M, P)_{R^{\prime}}$ with the $X^{\prime}$-grading:

$$
H\left(M,{ }_{R} P\right)_{x^{\prime}}=\left\{f \in \operatorname{Hom}_{(G, X, R)-g r}(M, P) \mid f(m) \subseteq P_{x^{\prime}}\right\} \quad\left(x^{\prime} \in X^{\prime}\right) .
$$

The functor $H(-, R P)$ associates to $f \in \operatorname{Hom}_{(G, X, R)-g r}(M, N)$, the map $H\left(f,_{R} P\right): H\left(N,{ }_{R} P\right) \rightarrow H_{R}(M, P)$, given by: $H\left(f,{ }_{R} P\right)(\varphi)=\varphi \circ f$ for all $\varphi \in H\left(N,{ }_{R} P\right)$.

It is left to the reader to check that the above three functors are well defined. Similarly there are two covariant functors $P \hat{\otimes}_{R^{\prime}}-$ : $\left(G^{\prime}, X^{\prime}, R^{\prime}\right)-g r \rightarrow(G, X, R)-g r$ and $H\left({ }_{R} P,-\right):(G, X, R)-g r \rightarrow$ $\left(G^{\prime}, X^{\prime}, R^{\prime}\right)-g r$ and a contravariant functor $H\left(-, P_{R^{\prime}}\right): g r-\left(R^{\prime}, X^{\prime}, G^{\prime}\right) \rightarrow$ $(G, X, R)-g r$.

If $\mathcal{C}$ is a category, then $\mathcal{C}^{\mathrm{op}}$ will denote the dual category.

Let $F: \mathcal{C} \rightarrow \mathcal{D}$ and $G: \mathcal{D} \rightarrow \mathcal{C}$ be two contravariant functors. Then we can consider $F: \mathcal{C} \rightarrow \mathcal{D}^{\text {op }}$ and $G: \mathcal{D}^{\text {op }} \rightarrow \mathcal{C}$ as covariant functors. We say that $(F, G)$ is a pair of adjoint contravariant functors on the right if $F: \mathcal{C} \rightarrow \mathcal{D}^{\text {op }}$ is left adjoint of $G: \mathcal{D}^{\text {op }} \rightarrow \mathcal{C}$.

Proposition 1.2. Let $P$ be an $R$ - $R^{\prime}$-graded bimodule.

(a) [M] The functor $-\hat{\otimes}_{R} P$ is left adjoint of $H\left(P_{R^{\prime}},-\right)$.

(b) $\left(H\left(-, R_{R} P\right), H\left(-, P_{R^{\prime}}\right)\right)$ is a pair of adjoint contravariant functors on the right.

Proof: See $[\mathbf{M}]$ for (a). In [MR] there is a proof of (b) in the particular case $G=X$ and $G^{\prime}=X^{\prime}$ with regular actions. The same method works in the gencral case.

For cvery $r \in R$ let $\lambda_{r}: R \rightarrow R$ be the left multiplication-by- $r$ map and $\chi_{r}: R \rightarrow R$ the right multiplication-by- $r$ map. The following lemma is obvious:

Lemma 1.3. Let $r \in R_{g}$ and $x \in X$. Then

(a) $\lambda_{r} \in \mathrm{Hom}_{g r-(R, X, G)}(R(x), R(g x))$.

(b) $\chi_{r} \in \operatorname{Hom}_{(G, X, R)-g r}((g x) R,(x) R)$. 
Definition 1.4. Let $F: g r-(R, X, G) \rightarrow g r-\left(R^{\prime}, X^{\prime}, G^{\prime}\right)$ be a covariant functor. The $X \times X^{\prime}$-graded $R$ - $R^{\prime}$-bimodule associated to $F$ is the right $R^{\prime}$-module $P=\underset{x \in X}{\oplus} F(R(x))$ with the following structure of left $R$-module:

$$
r p=\left[F\left(\lambda_{r}\right)\right](p) \quad\left(r \in R_{g}, p \in F(R(x))\right)
$$

and the $X \times X^{\prime}$-grading:

$$
P_{\left(x, x^{\prime}\right)}=F(R(x))_{x^{\prime}} \quad\left(x \in X, x^{\prime} \in X^{\prime}\right) .
$$

Let now $F:(G, X, R)-g r \rightarrow g r-\left(R^{\prime}, X^{\prime}, G^{\prime}\right)$ be a contravariant functor. The $X \times X^{\prime}$-graded $R-R^{\prime}$-bimolule associated to $F$ is the right $R^{\prime}$ module $P=\underset{x \in X}{\oplus} F((x) R)$ with the following structure of left $R$-module

$$
\left.r p=\left[F\left(\chi_{r}\right)\right](p) \quad\left(r \in R_{g}, p \in F((x) R)\right)\right)
$$

and the $X \times X^{\prime}$-grading

$$
P_{\left(x, x^{\prime}\right)}=F((x) R)_{x^{\prime}} \quad\left(x \in X, x^{\prime} \in X^{\prime}\right) .
$$

Lemma 1.5. Let $M \in(G, X, R)-g r$. Then $\operatorname{Hom}_{(G, X, R)-g r}((x) R, M)$ $\cong{ }_{x} M$ and this isomorphism is natural in each variable.

Proof: For any $m \in M$ let $\chi_{m}: R \rightarrow M$ be the right-multiplicationby-r map. For every $m \in M, \chi_{m} \in \operatorname{Hom}_{(G, X, R)-g r}\left((x) R,,_{R} M\right)$ if and only if $m \in{ }_{x} M$. Moreover, for every $f \in \operatorname{Hom}_{(G, X, R)-g_{r}}(R(x), P), f=$ $\chi_{f(1)}$. Therefore the map $m \mapsto \chi_{m}$ is an isomorphism between ${ }_{x} M$ and $\operatorname{Hom}_{(C, X, R)-g_{r}}((x R, M)$. The naturallity is left to the reader.

Now we are ready to describe all covariant and contravariant functors between categories of modules graded by $G$-sets.

Proposition 1.6. (a) $[\mathbf{M}] F: g r-(R, X, G) \rightarrow g r-\left(R^{\prime}, X^{\prime}, G^{\prime}\right)$ is a left adjoint functor if and only if there exists an $X \times X^{\prime}$-graded $R$ - $R^{\prime}$-bimodule $P$ such that $F \cong-\hat{\otimes}_{R} P$. Moreover, in this case $P$ is isomorphic to the $X \times X^{\prime}$-graded $R$ - $R^{\prime}$-bimodule associated to $F$.

(b) $F: g r-\left(R^{\prime}, X^{\prime}, G^{\prime}\right) \rightarrow g r-(R, X, G)$ is a right adjoint functor if and only if there exists an $X \times X^{\prime}$-graded $R$ - $R^{\prime}$-bimodule $P$ such that $F \cong H\left(P_{R^{\prime}},-\right)$.

(c) $F:(G, X, R)-g r \rightarrow g r-\left(R^{\prime}, X^{\prime}, G^{\prime}\right)^{\text {op }}$ is a left adjoint functor if and only if there exists an $X \times X^{\prime}$-graded $R$ - $R^{\prime}$-bimodule $P$ such that 
$F \cong H\left(-,{ }_{R} P\right)$. Moreover, in this case, $P$ is isomorphic to the $X \times X^{\prime}$. graded $R$ - $R^{\prime}$-bimodule associated to $F$.

Proof: (a) and (b) have been proved in [M]. For a proof of (c), an argument as in the proof of Proposition 2.1 of [MR] works, by using Lemma 1.5.

Now we focus our attention on some particular functors introduced in [M]. Some examples of these functors can be found in [D1], [D2], [NRV] and $[\mathrm{MN}]$.

Let $\rho: R \rightarrow R^{\prime}$ be a homomorphism of rings, $\xi: X \rightarrow X^{\prime}$ a map and $\gamma: G \rightarrow G^{\prime}$ a group homomorphism. Assume that the following conditions hold:

$$
\rho\left(R_{g}\right) \subseteq R_{\gamma(g)} \quad \xi(x g)=\xi(x) \gamma(g) \quad(x \in X, g \in G)
$$

Set $T=(\rho, \xi, \gamma)$ (see $[\mathrm{M}]$ ). For any $M \in g r-(R, X, G)$, let $T^{*}(M)=$ $M \otimes_{R} R^{\prime}$ and for every $x^{\prime} \in X^{\prime}$, let $T^{*}(M)_{x^{\prime}}$ be the additive subgroup of $T^{*}(M)$ generated by the clements of the form $m \otimes r^{\prime}$ with $m \in M_{x^{\prime}}: r \in$ $R_{g^{\prime}}^{\prime}$ and $\xi(x) g^{\prime}=x^{\prime}$.

For every $f \in \operatorname{Hom}_{g r-(R, X, G)}(M, N)$ set $T^{*}(f)=f \otimes R^{\prime}: T^{*}(M) \rightarrow$ $T^{*}(N)$.

Proposition 1.7. Let $T=(\rho, \xi, \gamma)$ and $T^{*}$ as above. The following ossertions hold:

(a) $T^{*}$ defines a left adjoint functor from $g r-(R, X, G)$ to $g^{r}-\left(R^{\prime}, X^{\prime}, G^{\prime}\right)$.

(b) The right adjoint functor of $T^{*}$ is isomorphic to the functor $T_{*}$ : $g r-\left(R^{\prime}, X^{\prime}, G^{\prime}\right) \rightarrow g r-(R, X, G)$ given as follows:

$$
T_{*}\left(M^{\prime}\right)=\underset{x \in X}{\oplus} T_{*}\left(M^{\prime}\right)_{x} \quad\left(M^{\prime} \in g r-\left(R^{\prime}, X^{\prime}, G^{\prime}\right)\right)
$$

where the map $m^{\prime} \mapsto m^{\prime 2}$ is an isomorphism of additive groups from $M_{\xi(x)}^{\prime}$ to $T_{*}\left(M^{\prime}\right)_{x}$ and the structure of right $R$-module on $T_{*}(M)$ is given by the following rule:

$$
\left(m^{\prime x}\right) r=\left(m^{\prime} \rho(r)\right)^{x g} \quad\left(m^{\prime} \in M^{\prime}, r \in R_{g}\right) .
$$

If $f \in \operatorname{Hom}_{g r-\left(R^{\prime} ; X^{\prime}, G^{\prime}\right)}\left(M^{\prime}, N^{\prime}\right)$ and $m^{\prime} \in M^{\prime}$ then $T_{*}(f)\left(m^{\prime 2}\right)=$ $f\left(m^{\prime}\right)^{x}$.

(c) $T_{*}$ is a left adjoint functor. 
Proof: (a) Clearly $T^{*}(M)=\sum_{x^{\prime} \in X^{\prime}} T^{*}(M)$ and $T^{*}(M)_{x^{\prime}} R_{g^{\prime}}^{\prime} \subseteq T^{*}(M)_{x^{\prime} g^{\prime}}$ for every $x^{\prime} \in X^{\prime}$ and $g^{\prime} \in G^{\prime}$.

Let $P=\underset{x \in X}{\oplus} R^{\prime}(\xi(x)) \in g r-\left(R^{\prime}, X^{\prime}, G^{\prime}\right)$. For any $x \in X$, let $u_{x}$ : $R^{\prime}(\xi(x)) \rightarrow P$ be the canonical monomorphism.

$P$ becomes an $X \times X^{\prime}$ graded $R$ - $R^{\prime}$-bimodule after the following rules:

$$
\begin{array}{ll}
r u_{x}\left(r^{\prime}\right)=u_{g x}\left(r r^{\prime}\right) & \left(g \in G, r \in G_{g}, r^{\prime} \in R^{\prime}, x \in X\right) \\
P_{\left(x, x^{\prime}\right)}=R^{\prime}(\xi(x))_{x^{\prime}} & \left(x \in X, x^{\prime} \in X^{\prime}\right) .
\end{array}
$$

Furthermore, for every $M \in g r-(R, X, G)$, the map

$$
\begin{aligned}
\phi_{M}: T^{*}(M) & \longrightarrow M \hat{\otimes}_{R} P \\
m \otimes r^{\prime} & \mapsto \sum_{x \in X} m_{x} \otimes u_{x}\left(r^{\prime}\right)
\end{aligned}
$$

is an isomorphism of right $R^{\prime}$-modules and for every $x^{\prime} \in X^{\prime}, \phi_{M}\left(T^{*}(M)_{x^{\prime}}\right)$ $=M \hat{\otimes}_{R} P_{x^{\prime}}$. Therefore, $T^{*}(M)=\underset{x^{\prime} \in X^{\prime}}{\oplus} T^{*}(M)_{x^{\prime}}$ and hence $T^{*}$ defines a functor from $g r-(R, X, G)$ to $g r-\left(R^{\prime}, X^{\prime}, G^{\prime}\right)$. Moreover $T^{*}$ is a left adjoint, because $T^{*} \cong-\hat{\otimes}_{R} P$.

(b) For every $M^{\prime} \in g r-\left(R^{\prime}, X^{\prime}, G^{\prime}\right)$,

$$
\begin{aligned}
H\left(P_{R^{\prime}}, M^{\prime}\right) & =\underset{x \in X}{\oplus} \operatorname{Hom}_{g r-\left(R^{\prime}, X^{\prime}, G^{\prime}\right)}\left(x P, M^{\prime}\right) \\
& =\underset{x \in X}{\oplus} \operatorname{Hom}_{g r-\left(R^{\prime}, X^{\prime}, G^{\prime}\right)}\left(R^{\prime}(\xi(x)), M^{\prime}\right) \cong \underset{x \in X}{\oplus} M_{\xi(x)}^{\prime} .
\end{aligned}
$$

Using this isomorphism it is straightforward to see that $H\left(P_{R^{\prime}},-\right) \cong$ $T_{*}$ and (b) follows from Proposition 1.2.

(c) Let $Q$ be the $X^{\prime} \times X$-graded $R^{\prime}$-R-bimodule associated to $T_{*}$.

For any $M^{\prime} \in g r-\left(R^{\prime}, X^{\prime}, G^{\prime}\right)$ we define

$$
\begin{aligned}
\Phi_{M^{\prime}}: M^{\prime} \hat{\otimes}_{R^{\prime}} Q \longrightarrow T_{*}\left(M^{\prime}\right) \\
m^{\prime} \otimes q \mapsto\left(m^{\prime} q\right)^{x} \quad\left(m^{\prime} \in M_{x^{\prime}}^{\prime}, q \in Q_{\left(x^{\prime}, x\right)}\right) .
\end{aligned}
$$

Then $\Phi_{M^{\prime}}$ is bijective and $\Phi$ defines an isomorphism of functors between $-\hat{\otimes}_{R^{\prime}} Q$ and $T_{*}$. Therefore $T_{*}$ is a left adjoint and $H\left(Q_{R^{\prime}}\right)$ is its right adjoint. 
Lemma 1.8. Let $T=(\rho, \xi, \gamma)$ as above and assume that $R^{\prime}=R$ and $\rho$ is the identity map. Consider $X^{\prime}$ as a right $G$-set by $x^{\prime} g=x^{\prime} \gamma(g)\left(x^{\prime} \in\right.$ $\left.X^{\prime}, g \in G\right)$. Then

(a) $g r-\left(R, X^{\prime}, G^{\prime}\right)=g r-\left(R, X^{\prime}, G\right)$.

(b) $T^{*}=S^{*}$ and $T_{*}=S_{*}$ where $S=\left(1_{R}, \xi, 1_{G}\right)$.

Proof: (a) Clearly an $M \in g r-\left(R, X^{\prime}, G^{\prime}\right)$ is an object of $g r-$ $\left(R, X^{\prime}, G\right)$ because for every $x^{\prime} \in X^{\prime}, M_{x^{\prime}} R_{g} \subseteq M_{x^{\prime}} R_{\gamma(g)} \subseteq M_{x^{\prime} \gamma(g)}=$ $M_{x^{\prime} g}$.

On the other hand, let $M \in g r-\left(R, X^{\prime}, G\right)$. Let $g^{\prime} \in G^{\prime}$ and $0 \neq r \in$ $R_{g^{\prime}}$. If $g \in \operatorname{Supp}_{R}(r)$, then $r_{g}=\rho\left(r_{g}\right) \in R_{\gamma(g)}$. Thus $r_{g}=\left(r_{g}\right)_{\gamma(g)}$. If $\gamma(g) \neq g^{\prime}$, then

$$
0=r_{\gamma(g)}=\sum_{h \in \operatorname{Supp}_{R}(r) \cap \gamma^{-1}(\gamma(g))}\left(r_{h}\right)_{\gamma(g)}=\sum_{h \in \operatorname{Supp}_{R}(r) \cap \gamma^{-1}(\gamma(g))} r_{h} .
$$

Therefore $r_{g}=0$. Thus $\operatorname{Supp}_{R}(r) \subseteq \gamma^{-1}\left(g^{\prime}\right)$. Let now $x^{\prime} \in X^{\prime}$. Then $M_{x^{\prime}} r=M_{x^{\prime}} \sum_{g \in \operatorname{Supp}_{R}(r)} r_{g} \subseteq \sum_{g \in \operatorname{Supp}_{R}(r)} M_{x^{\prime} g}=\sum_{g \in \operatorname{Supp}_{R}(r)} M_{x^{\prime} \gamma(g)}=M_{x^{\prime} g^{\prime}}$.

(b) Is obvious.

Let $R$ be a $G$-graded ring and $\xi: X \rightarrow X^{\prime}$ a morphism of right $G$ sets. If $T=\left(1_{R}, \xi, 1_{G}\right)$, then the functors $T^{*}$ and $T_{*}$ are the functors $T_{\xi}$ and $S^{\xi}$ defined in [NRV]. In [NRV] the anthors prove that if for every $x^{\prime} \in X^{\prime}, \xi^{-1}\left(x^{\prime}\right)$ is finite, then $S^{\xi}$ is left adjoint of $T_{\xi}$. But the converse is also true as the following proposition shows.

Proposition 1.9. Let $R$ be a $G$-graded ring and $\xi: X \rightarrow X^{\prime}$ a morphism of right $G$-sets. Let $F$ be the right adjoint functor of $S^{\xi} . F$ is a left adjoint functor if and only if for every $x^{\prime} \in X^{\prime}, \xi^{-1}\left(x^{\prime}\right)$ is finite. Moreover, in this case $F$ is isomorphic to $T_{\xi}$.

Proof: Let $Q$ be the $X^{\prime} \times X$-graded $R$ - $R$-bimodule associated to $S^{\xi}$. By Proposition 1.6, $S^{\xi} \cong-\hat{\otimes}_{R} Q$ and $F \cong H\left(Q_{R^{\prime}}-\right.$.

Assume that $\xi^{-1}\left(x^{\prime}\right)$ is finite for every $x^{\prime} \in X^{\prime}$. For each $M \in g^{r}-(R, X, G)$, let

$$
\begin{aligned}
& \mu_{M}: H\left(Q_{R}, M\right) \longrightarrow T_{\xi}(M) \\
& \mu_{M}(f)=\sum_{\xi(x)=x^{\prime}}\left(f\left(1^{x}\right)\right)_{x} \quad\left(f \in H\left(Q_{R^{\prime}} M\right)_{x^{\prime}}\right) .
\end{aligned}
$$

The claim follows by proving that $\mu: H\left(Q_{R},-\right) \rightarrow T_{\xi}$ is a functorial isomorphism. This is left to the reader. 
Conversely, if $H\left(Q_{R},-\right)$ is a left adjoint functor, then it preserves direct sums. Let $x^{\prime} \in X^{\prime}$. Consider the functor $(-)_{x^{\prime}}: g r-\left(R^{\prime}, X^{\prime}, G^{\prime}\right) \rightarrow$ $\bmod -R_{c}^{\prime}$ which associates to $M^{\prime} \in g r-\left(R^{\prime}, X^{\prime}, G^{\prime}\right)$ the right $R_{e}^{\prime}$-module $M_{x^{\prime}}^{\prime}$ and to $f \in \operatorname{Hom}_{g^{r}-\left(R^{\prime}, X^{\prime}, G^{\prime}\right)}\left(M^{\prime}, N^{\prime}\right)$ the map $f_{x^{\prime}}: m^{\prime} \mapsto f\left(m_{x^{\prime}}^{\prime}\right)$. This functor preserves direct sums and hence $\operatorname{Hom}_{g r-(R, X, G)}\left(x_{x^{\prime}} Q,-\right) \cong$ $(-)_{\pi^{\prime}} \circ H\left(Q_{R},-\right)$ preserves direct sums too. This implies that ${ }_{x^{\prime}} Q=$ $S_{\xi}\left(R^{\prime}\left(x^{\prime}\right)\right)$ is finitely generated (cf. [S]).

Let $\alpha=\sum_{\xi(x)=x^{\prime}}\left(1^{x}\right) r_{x}=0$ with $r_{x} \in R$. For every $y \in X, \alpha_{y}=$ $\sum_{\xi(x)=x^{\prime}}\left(1^{x}\right)\left(\sum_{x g=y}^{\xi(x)}\left(r_{x}\right)_{g}\right)=0$. Therefore, we can assume, without loss of generality, that there exists $y \in Y$ such that for every $g \in \operatorname{Supp}\left(r_{x}\right), x g=$ $y$. Thus $\left(\sum_{\xi(x)=x^{\prime}} r_{x}\right)^{y}=\alpha_{y}=0$ and hence $\sum_{\xi(x)=x^{\prime}} r_{x}=0$. But $r_{x}$ has degree $x$ in $(y) R$. We conclude that $r_{x}=0$ for every $x \in X$. Therefore the sum $\sum_{x \in X} 1^{x} R$ is direct.

On the other hand, if $r \in R_{g} \cap Q_{\left(x^{\prime}, x\right)}$, then $x^{\prime} g=\xi(x)$. Therefore $r^{x}=1^{x g^{-1}} r$. We conclude that $x^{\prime} Q=\underset{\xi(x)=x^{\prime}}{\ominus} 1^{x} R$. Since $x^{\prime} Q$ is finitely generated, the sum is finite, i.e. $\xi^{-1}\left(x^{\prime}\right)$ is finite.

\section{Equivalences and Morita dualities}

Propositions 1.2 and 1.6 set up the framework to give a description of all the equivalences and Morita dualities (in the sense of Colby-Fuller [CF]) between categories of modules graded by $G$-sets. This section is dedicated to present that description. Since the methods are similar to those given in [Rí2] and [MR] we shall skip the proofs.

Let $\hat{R}$ be the $X \times X$-graded $R$-R-bimodule associated to the identity functor in $g r-(R, G, X)$.

Let $P$ be an $X \times X^{\prime}$-graded $R$ - $R^{\prime}$-bimodule and $Q$ an $X^{\prime} \times X^{\prime \prime}$-graded $R^{\prime}$ - $R^{\prime \prime}$-bimodule. Then $P \hat{\otimes}_{R^{\prime}} Q$ is a subbimodule of $P \otimes_{R} Q$ and $\left(P \hat{\otimes}_{R^{\prime}}\right.$ $Q)_{\left(x, x^{\prime \prime}\right)}={ }_{x} P \hat{\otimes}_{R} Q_{x^{\prime \prime}}\left(x \in X, x^{\prime \prime} \in X^{\prime \prime}\right)$ defines an $X \times X^{\prime \prime}$-grading on ${ }_{R} P \hat{\otimes}_{R^{\prime}} Q_{R^{\prime \prime}}$. Furthermore the functors $\left(-\hat{\otimes}_{R^{\prime}} Q\right) \circ\left(-\hat{\otimes}_{R} P\right)$ and $-\hat{\otimes}_{R}\left(P \hat{\otimes}_{R^{\prime}} Q\right)$ are isomorphic.

Proposition 2.1. The following assertions are equivalent:

(a) The categories $g r-(R, X, G)$ and $g r-\left(R^{\prime}, X^{\prime}, G^{\prime}\right)$ are equivalent.

(b) There are an $X \times X^{\prime}$-graded $R-R^{\prime}$-bimodule $P$ and an $X^{\prime} \times X$ graded $R^{\prime}-R$-bimodule $Q$ such that $\hat{R} \cong P \hat{\otimes}_{R^{\prime}} Q$ and $\hat{R} \cong Q \hat{\otimes}_{R} P$ as bigroded bimodules. 
Moreover, if $P$ and $Q$ are as in (b), then $-\hat{\otimes}_{R} P$ and $-\hat{\otimes}_{R^{\prime}} Q$ are inverse equivalences of categories.

Corollary 2.2. (a) The categories $g r-(R, X, G)$ and $g r-\left(R^{\prime}, X^{\prime}, G^{\prime}\right)$ are equivalent if and only if the categories $(G, X, R)-g r$ and $\left(G^{\prime}, X^{\prime}, R^{\prime}\right)-g r$ are equivalent.

(b) Let $P$ be an $X \times X^{\prime}$-graded $R$ - $R^{\prime}$-bimodule. $-\hat{\otimes}_{R} P$ : $g r-(R, X, G) \rightarrow g r-\left(R^{\prime}, X^{\prime}, G^{\prime}\right)$ is an equivalence if and only if $P \hat{\otimes}_{R^{\prime}}-:\left(G^{\prime}, X^{\prime}, R^{\prime}\right)-g r \rightarrow(G, X, R)-g r$ is an equivalence.

Definition. Let $P$ be an $X \times X^{\prime}$-graded $R$ - $R^{\prime}$-bimodule and $Q$ an $X \times X^{\prime \prime}$-graded $R$ - $R^{\prime \prime}$-bimodule. We define

$$
H\left({ }_{R} P,{ }_{R} Q\right)=H\left({ }_{R} P, Q\right) \cap H\left(P,{ }_{R} Q\right) .
$$

If we consider $\operatorname{Hom}_{R}(P, Q)$ as an $R^{\prime}$ - $R^{\prime \prime}$-bimodule by

$$
\left(r^{\prime} f r^{\prime \prime}\right)(p)=f\left(p r^{\prime}\right) r^{\prime \prime} \quad\left(f \in \operatorname{Hom}_{R}(P, Q), p \in P, r^{\prime} \in R^{\prime}, r^{\prime \prime} \in R^{\prime \prime}\right)
$$

then $H_{R}(P, Q)$ is a subbimodule of ${ }_{R^{\prime}} \operatorname{Hom}_{R}(P, Q)_{R^{\prime \prime}}$, and it is an $X^{\prime} \times X^{\prime \prime}$-graded $R^{\prime}$ - $R^{\prime \prime}$-bimodule with the following grading:

$$
H\left({ }_{R} P,{ }_{R} Q\right)_{\left(x^{\prime}, x^{\prime \prime}\right)}={ }_{x^{\prime}} H\left({ }_{R} P, Q\right) \cap H\left(P,{ }_{R} Q\right)_{x^{\prime \prime}} \quad\left(x^{\prime} \in X^{\prime} ; x^{\prime \prime} \in X^{\prime \prime}\right) .
$$

Similarly, if $P$ is an $X^{\prime} \times X$-graded $R^{\prime}$-R-bimodule and $Q$ is an $X^{\prime \prime} \times X$ graded $R^{\prime \prime}$-R-bimodule, then $H\left(P_{R}, Q_{R}\right)=H\left(P_{R}, Q\right) \cap H\left(P, Q_{R}\right)$ has a natural structure of $X^{\prime \prime} \times X^{\prime}$-graded $R^{\prime \prime}$ - $R^{\prime}$-bimodule.

Let $P$ be an $X \times X^{\prime}$-graded $R$ - $R^{\prime}$-bimodule. Consider the following map:

$$
\begin{aligned}
\chi^{P}: & \hat{R}^{\prime} \longrightarrow H\left({ }_{R} P,{ }_{R} P\right) \\
& \chi^{P}(r)(p)=\left(p r^{\prime}\right)_{y^{\prime}} \quad\left(r^{\prime} \in R^{\prime}\left(x^{\prime}\right)_{y^{\prime}}, p \in P\right)
\end{aligned}
$$

$\chi^{P}$ is a homomorphism of $X^{\prime} \times X^{\prime}$-graded $R^{\prime} \times R^{\prime}$-bimodules.

Similarly there is an $X \times X$-graded homomorphism of $R$ - $R$-bimodules

$$
\begin{aligned}
\lambda^{P}: \hat{R} \longrightarrow H\left(P_{R^{\prime}}, P_{R^{\prime}}\right) & \\
& \lambda^{P}(r)(p)={ }_{x}(r p) \quad(r \in R(x), p \in P) .
\end{aligned}
$$

Theorem 2.3. Let $P$ be an $X \times X^{\prime}$-graded $R$ - $R^{\prime}$-bimodule. The following conditions are equivalent:

(a) The functor $-\hat{\otimes}_{R} P: g r-(R, X, G) \rightarrow g r-\left(R^{\prime}, X^{\prime}, G^{\prime}\right)$ is an equivalence of categories. 
(b) The functor $H\left(P_{R^{\prime}},-\right): g r-\left(R^{\prime}, X^{\prime}, G^{\prime \prime}\right) \rightarrow g r-(R, X, G)$ is an equivalence of categories.

(c) $P_{R^{\prime}}$ is a projective generalor in $g r-\left(R^{\prime}, X^{\prime}, G^{\prime}\right), \lambda^{P}: \hat{R} \rightarrow$ $H\left(P_{R^{\prime}}, P_{R^{\prime}}\right)$ is an isomorphism and for every $x \in X,{ }_{x} P_{R^{\prime}}$ is finitely generated.

(d) ${ }_{R}^{P}$ is a projective generalor in $(G, X, R)-g r, \chi^{P}: \hat{R}^{\prime} \rightarrow$ $H\left({ }_{R} P_{R} P\right)$ is an isomorphism and, for every $x^{\prime} \in X^{\prime},{ }_{R} P_{x^{\prime}}$ is finitely generated.

Let $A$ be a ring and $G$ a group. For any $G$-grading in $A$, the category mod- $A$ can be secn as a category of $X$-graded right $A$-modules where $X$ is a singleton. I ast theorem yields to the following two corollaries.

Corollary 2.4. Let $R$ be a $G$-graded ring, $X$ a right $G$-set and $A$ a ring. The following are equivolent.

(a) $g r-(R, X, G)$ is equivalent to $\bmod -A$.

(b) $(G, X, R)-g r$ is equivalent to $A$-mod.

(c) There exists a finitely generated projective generator $P$ in $g r-(R, X, G)$ such that $A$ is isomorphic to $\operatorname{End}_{g r-(R, X, G)}(P)$.

(d) There exists a finite subset $F$ of $X$ such thot $U=\underset{x \in F}{\bigoplus} R(x)$ is a generator of $g r-(R, X, G)$ and $\mathrm{Fnd}_{g r-(R, X, G)}(U)$ is Morita equivalent to $A$.

Corollary 2.5. Let $R$ be a $G$-graded ring and $X$ a right $G$-set. The following are equivalent.

(a) There exists a ring $A$ such that $g r-(R, X, G)$ is equivalent to $\bmod -A$.

(b) There exists a $\operatorname{ring} A$ such that $(G, X, R)-g r$ is equivalent to $A$-rnod.

(c) $g r-(R, X, G)$ has a finitely generated (projective) generator.

(d) There exists a finite subset $F$ of $X$ such that $\underset{x \in F}{\oplus} R(x)$ is a generator of $g r-(R, X, G)$.

Example 2.6. Consider $T=(\rho, \xi, \gamma)$ as in Section 1. The functor $T^{*}$ is isomorphic to $-\hat{\otimes}_{R} P$ where $P=\underset{x \in X}{\oplus_{X}} R^{\prime}(\xi(x)$ ) (see the proof of Proposition 1.7). Since for cvery $x \in X,{ }_{x} P_{R^{\prime}}=R^{\prime}(\xi(x))$ is finitely generated and projective, $T^{*}$ is an equivalence if and only if $P_{R^{\prime}}$ generates $g r-\left(R^{\prime}, X^{\prime}, G^{\prime}\right)$ and $\lambda^{P}: \hat{R} \rightarrow H\left(P_{R^{\prime}}, P_{R^{\prime}}\right)$ is an isomorphism. We claim that $T^{*} \cong-\otimes_{R} R^{\prime}: g r-(R, X, G) \rightarrow g^{r}-\left(R^{\prime}, X^{\prime}, G^{\prime}\right)$ is an equivalence if and only if the following conditions hold

(a) $\rho$ is an isomorphism between $R$ and $\underset{\operatorname{Im}(\xi) g^{\prime} \sim \operatorname{Im}(\xi) \neq ?}{\ominus} R_{g^{\prime}}^{\prime}$ 
(b) For every $x^{\prime} \in X^{\prime}$ there exists $r_{1}^{\prime} \in R_{g_{2}^{\prime}}^{\prime}, r_{2} \in R_{g_{2}^{\prime}}^{\prime}, \ldots, r_{n}^{\prime} \in$ $R_{g_{n}^{\prime}}^{\prime} ; s_{1}^{\prime} \in R_{g_{2}^{\prime}-1}^{\prime}, s_{2}^{\prime} \in R_{g_{2}^{\prime}-1}^{\prime}, \ldots, s_{n}^{\prime} \in R_{g_{n}^{\prime}-1}^{\prime}$, such that $x^{\prime} g_{i}^{\prime} \in$ $\operatorname{Im}(\xi)$ for every $i=1,2, \ldots, n$ and $\sum_{i=1}^{n} r_{1}^{\prime} s_{1}^{\prime}=1$.

Proof; If $r^{\prime} \in R^{\prime}$ and $x \in X$, then $r^{\prime x}$ will denote $r^{\prime}$ considered in $R^{\prime}(\xi(x))$.

Assume $T^{*}$ is an equivalence. Then $\lambda^{P}: \hat{R} \rightarrow H\left(P_{R^{\prime}}, P_{R^{\prime}}\right)$ is an ismorphism and $P_{R^{\prime}}$ is a gencrator. Let $r \in \operatorname{Ker}(\rho)$. For every $p \in P,\left[\lambda^{P}\left(r^{x}\right)\right](p)={ }_{x}(r p)={ }_{x}(\rho(r) p)=0$. Thus $r^{x} \in \operatorname{Ker}\left(\lambda^{P}\right)=0$. Thercforc $\rho$ is injective. If $r \in R_{g}$ and $x \in X$, then $\rho(r) \in R_{\gamma(g)}$ and $\xi(x) \gamma(g)=\xi(x g)$. Therefore $\rho(R) \subseteq \underset{\operatorname{Im}(\xi) g^{\prime} \cap \operatorname{Im}(\xi) \neq ?}{\oplus} R_{g^{\prime}}^{\prime}$. On the other hand, let $r^{\prime} \in R_{g^{\prime}}^{\prime}$ and $x, y \in X$ such that $\xi(x) g^{\prime}=\xi(y)$. Consider the following homomorphism of right $R^{\prime}$-modules

$$
\begin{aligned}
L: P \longrightarrow P & \longrightarrow \\
L\left(s^{\prime z}\right)= & \left\{\begin{array}{ll}
\left(r^{\prime} s^{\prime}\right)^{x} & \text { if } z=y \\
0 & \text { if } z \neq x
\end{array} \quad\left(s^{\prime} \in R^{\prime}, z \in X\right) .\right.
\end{aligned}
$$

Plainly $L \in H\left(P_{R^{\prime}}, P_{R^{\prime}}\right)_{(x, y)}$, thus there exists $r \in R(x)_{y}$ such that $L=\lambda^{P}\left(r^{x}\right)$. But $r^{\prime x}=L\left(1^{y}\right)=\lambda^{P}(r)\left(1^{y}\right)={ }_{x}\left(\rho(r)^{x}\right)=\rho(r)^{x}$. This proves (a).

Let $x^{\prime} \in X^{\prime}$. Since $P_{R^{\prime}}=\underset{x \in X}{\oplus} R^{\prime}(\xi(x))$ is a generator of $g r-\left(R^{\prime}, X^{\prime}, G^{\prime}\right)$, then $\left\{R^{\prime}(\xi(x)) \mid x \in X\right\}$ is a set of generators of $g r-\left(R^{\prime}, X^{\prime}, G^{\prime}\right)$. Therefore there exist $x_{1}, x_{2}, \ldots, x_{n} \in X$ and an $X^{\prime}$. graded epimorphism $\stackrel{n}{\oplus} R^{\prime}\left(\xi\left(x_{1}\right)\right) \rightarrow R^{\prime}\left(x^{\prime}\right)$. Thus there exist $r_{1}^{\prime} \in$ $R^{\prime}\left(x^{\prime}\right)_{\xi\left(x_{1}\right)}, r_{2}^{\prime} \in R^{\prime}\left(x^{\prime}\right)_{\xi\left(x_{2}\right)}, \ldots, r_{n}^{\prime} \in R^{\prime}\left(x^{\prime}\right)_{\xi\left(x_{n}\right)}$ and $s_{1}^{\prime} \in R^{\prime}\left(\xi\left(x_{1}\right)\right)_{x^{\prime}}$, $s_{2}^{\prime} \in R^{\prime}\left(\xi\left(x_{2}\right)\right)_{x^{\prime}}, \ldots, s_{n}^{\prime} \in R^{\prime}\left(\xi\left(x_{n}\right)\right)_{x^{\prime}}$ such that $\sum_{i=1}^{n} r_{1}^{\prime} s_{1}^{\prime}=1$. If we replace the $s_{1}^{\prime}$ 's and the $r_{i}^{\prime}$ 's by its homogeneous components we can assume the they are homogencous. Moreover, if we take the homogeneous component of degree 1 we may assume that $r_{1}^{\prime}$ and $s_{1}^{\prime}$ have inverse degrees and if $r_{1}^{\prime}$ has degree $g_{1}^{\prime}$, then $x^{\prime} g_{1}^{\prime}=\xi\left(x_{1}\right)$. This proves (b).

Conversely, assume that (a) and (b) hold. Since $\lambda^{P}$ is an $X \times X$-graded homomorphism, to prove that it is injective it is enought to prove that if $0 \neq r \in R(x)_{y}=P_{(x, y)}$, then $\lambda^{P}\left(r^{x}\right) \neq 0$. But $x g=y$ for every $g \in \operatorname{Supp}(r)$. Thereforc $r 1^{y}=\sum_{g \in \operatorname{Supp}(r)} r^{g y}=r^{x}$. Thus $\left[\lambda^{P}\left(r^{x}\right)\right]\left(1^{y}\right)=$ ${ }_{x}\left(r 1^{y}\right)={ }_{x}\left(r^{x}\right) \neq 0$. Because $\lambda^{P}$ is $X \times X$-graded, to prove that it is surjective it is enough to see that $H\left(P_{R^{\prime}}, P_{R^{\prime}}\right)_{(x ; y)} \subseteq \operatorname{Im}\left(\lambda^{P}\right)$ for every 
$(x, y) \in X \times X$. Let $f \in H\left(P_{R^{\prime}}, P_{R^{\prime}}\right)_{(x, y)}$ and $r^{\prime}=f\left(1^{y}\right) \in R^{\prime}(\xi(x))_{\xi(y)}$. For every $g^{\prime} \in \operatorname{Supp}\left(r^{\prime}\right), \xi(x) g^{\prime}=\xi(y)$. Therefore, by (a) there exists $r_{g^{\prime}} \in R(x)_{y}$ such that $\rho\left(r_{g^{\prime}}\right)=r_{g^{\prime}}^{\prime}$. Then it is easy to see that $f=\lambda^{P}(r)$ where $r=\sum_{g^{\prime} \in \operatorname{Supp}\left(r^{\prime}\right)} r_{g^{\prime}}$.

Let $x^{\prime} \in X^{\prime}$. Let $r_{i}^{\prime}, s_{i}^{\prime}$ and $g_{i}^{\prime}(i=1,2, \ldots, n)$ as in (b). Let $L$ : $\stackrel{\oplus}{n}_{i=1}^{n} R^{\prime}\left(x^{\prime} g_{1}^{\prime}\right) \rightarrow R^{\prime}\left(x^{\prime}\right)$ given by $L\left(a_{1}^{\prime}, a_{2}^{\prime}, \ldots, a_{n}^{\prime}\right)=\sum_{i=1}^{n} r_{1}^{\prime} a_{1}^{\prime} . \quad L$ is an homomorphism of $X^{\prime}$-graded modules and $1=L\left(r_{1}^{\prime}, r_{2}^{\prime}, \ldots, r_{n}^{\prime}\right)$. But $x^{\prime} g_{1}^{\prime} \in \operatorname{Im}(\xi)$. Therefore $\left\{R^{\prime}(\xi(x)) \mid x \in X\right\}$ is a set of generators of $g r-\left(R^{\prime}, X^{\prime}, G^{\prime}\right)$. Therefore $P_{R^{\prime}}$ is a generator of $g r-\left(R^{\prime}, X^{\prime}, G^{\prime}\right)$. This finish the proof.

Example 2.7. As a particular case of Example 2.6, let $R$ be a $G$ graded ring, $X$ a right $G$-set, $x \in X$ and $G_{x}$ the stabilizer of $x$ in $G$. Consider $Y=\{x\}$ as a right $G_{x}$-set. Set $T=(\rho, \xi, \gamma)$ where $\rho: R_{G_{x}} \rightarrow$ $R, \xi: Y \rightarrow X$ and $\gamma: H \rightarrow G$ are inclusion maps.

In this case condition (a) in the above example always holds. On the other hand $g r-\left(R_{H}, Y, H\right)=\bmod -R_{H}$ and the functor $T_{*}$ is isomorphic to the functor $(-)_{x}$ which associates $M \in g r-(R, X, G)$ to $M_{x}$ and acts on morphisms by restricting on the domains. In this situation the result proved in last example translates into:

The following conditions are equivalent:

(a) $-\otimes_{R_{G_{x}}} R:$ mod $-R_{G_{x}} \rightarrow g r-(R, X, G)$ is an cquivalence.

(b) $(-)_{x}$ is an equivalence.

(c) $R(x)$ is a generator in $g r-(R, X, G)$.

(d) For every $y \in Y, R_{x^{-1} x}=R_{x^{-1} y} \cdot R_{y^{-1} x}$ (where $R_{x^{-1} y}=\underset{x y=y}{\oplus} R_{g}$ ).

Notice that condition (d) implies that $G$ acts transitively on $X$.

When $X=G$ with regular right action, the above cquivalent conditions do not depend on $x$. Namely, if $R(g)$ is a generator in $g r-R$, then for every $x \in G, R(x)$ is a generator too. Graded rings satisfying these condition are the strongly graded rings. When $X$ is not $G$ the situation is not so symmetric, as the following example shows.

Exampie 2.8. Let $A$ be a ring and $B$ a proper overring such that the inclusion map $j: A \rightarrow B$ splits as a morphism of $A-A$-bimodules, i.e. there is an $A$ - $A$-bimodule homomorphism $\varepsilon: A \rightarrow A$ such that $\varepsilon \circ j=1_{A}$.

Let $G$ be the permutation group on three elements. Let $a \in G$ be a transposition and $b \in G$ a 3-cycle. Let $K$ be the subgronp of $G$ generated by $b$ and $H$ the subgroup of $G$ generated by $a$. 
Let $M=A \times B \times B$ considered as an $A$ - $A$-bimodule. If we define

$$
\left.\begin{array}{l}
b(x, y, z)=(\varepsilon(z), \varepsilon(x), \varepsilon(y)) \\
(x, y, z) b=(\varepsilon(y), \varepsilon(z), \varepsilon(x))
\end{array}\right\}(x, y, z) \in M
$$

then $M$ becomes an $A[K] \times A[K]$-bimodule. Consider $R=A[K] \mathrm{n} M$ the trivial extension of $A[K]$ by $M$. The elements of $R$ are pairs $(\alpha, m)$ with $\alpha \in A[K]$ and $m \in M$ with the ring structure given by

$$
\left.\begin{array}{l}
(\alpha, m)+(\beta, n)=(\alpha+\beta, m+n) \\
(\alpha, m)(\beta, n)=(\alpha \beta, \alpha n+m \beta)
\end{array}\right\} \alpha, \beta \in A[K], m, n \in M
$$

Consider in $R$ the following $G$-grading:

$$
\begin{array}{lll}
R_{e}=A \times 0 & R_{b}=A b \times 0 & R_{b^{2}}=A b^{2} \times 0 \\
R_{a}=0 \times(A \times 0 \times 0) & R_{a b}=0 \times(0 \times B \times 0) & R_{a b^{2}}=0 \times(0 \times 0 \times B) .
\end{array}
$$

Let $X$ be the set of right $H$-cosets and consider $G$ acting on $X$ by right translation. Let $x=H$ and $y=H b$. Then

$$
\begin{aligned}
R_{x^{-1} x}=R_{H} & =R_{H b} \cdot R_{b^{-1} H}=R_{x^{-1} y} \cdot R_{y^{-1} x} \\
R_{y^{-1} x} \cdot R_{x^{-1} y} & =R_{b^{-1} H} \cdot R_{H b}=\left[A b^{2} \times(0 \times B \times 0)\right][A b \times(0 \times B \times 0)]= \\
& =A \times(0 \times 0 \times A) \neq A \times(0 \times 0 \times B)=R_{b^{-1} H b}=R_{y^{-1} y} .
\end{aligned}
$$

Therefore, by Example 2.7, $R(x)$ is a generator in $g r-(R, X, G)$ while? $R(y)$ is not.

In [CF] the authors extend the concept of Morita duality to Grothendieck categories. Let $(F: \mathcal{C} \rightarrow \mathcal{D}, G: \mathcal{D} \rightarrow \mathcal{C})$ be a pair of adjoint functors on the right. Let us denote by $\sigma: 1_{C} \rightarrow G F$ and $\tau: 1_{\mathcal{D}} \rightarrow F G$ the units of the adjonction (they can be interpreted as unit and counit of a pair of covariant functors). Let

$$
\begin{aligned}
\mathcal{R}_{\mathcal{C}} & =\left\{C \in \mathcal{C} \mid \sigma_{C} \text { is an isomorphism }\right\} \\
\mathcal{R}_{\mathcal{D}} & =\left\{D \in \mathcal{D} \mid \sigma_{\mathcal{D}} \text { is an isomorphism }\right\} .
\end{aligned}
$$

Then $F$ and $G$ induce a duality between $\mathcal{R}_{C}$ and $\mathcal{R}_{\mathcal{D}}$.

We say that the pair $(F ; G)$ is a Morita Duality if $F$ and $G$ are exact and the subcategories $\mathcal{R}_{\mathcal{C}}$ and $\mathcal{R}_{\mathcal{D}}$ contain a set of generators of $\mathcal{C}$ and $\mathcal{D}$, respectively, and both are closed under subobjects and quotient objects.

In [AW] the authors define an alternative version of Morita duality for Grothendieck categories which is not equivalent to the Colby-Fuller's 
one in general but it is so in the case of categories of modules graded by $G$-sets (see [GG]).

Note that if $(F, G)$ is a Morita duality, then $\mathcal{R}_{C}$ and $\mathcal{R}_{\mathcal{D}}$ are closed under finite coproducts, therefore $\mathcal{R}_{\mathcal{C}}$ and $\mathcal{R}_{\mathcal{D}}$ contain any finitely generated object of $\mathcal{C}$ and $\mathcal{D}$, respectively,

Let $P$ be is a $X \times X^{\prime}$-graded $R-R^{\prime}$-bimodule. Let $u: 1_{g r-(R, X, G)} \rightarrow$ $H\left(P_{R^{\prime}},-\right) \circ\left(-\hat{\otimes}_{R} P\right)$ and $c:\left(-\hat{\otimes}_{R} P\right) \circ H\left(P_{R^{\prime}},-\right) \rightarrow 1_{g r-\left(R^{\prime}, X^{\prime}, G^{\prime}\right)}$ the unit and the counit of the adjonction pair $\left(H\left(P_{R^{\prime}},-\right),\left(-\hat{\otimes}_{R} P\right)\right)$ and $\sigma: 1_{(G, X, R)-g \tau} \rightarrow H\left(-, P_{R^{\prime}}\right) \circ H\left(-, R_{R} P\right)$ and $\tau: 1_{g r-\left(R^{\prime}, X^{\prime}, G^{\prime}\right)} \rightarrow$ $H\left(-,{ }_{R} P\right) \circ H\left(-, P_{R^{\prime}}\right)$ the unit and counit of the adjonction pair $\left(H\left(-,{ }_{R} P\right), H\left(-, P_{R^{\prime}}\right)\right)$.

An object $M \in(G, X, R)-g r$ (resp. $\left.N \in g r-\left(R^{\prime}, X^{\prime}, G^{\prime}\right)\right)$ is said to be $P$-reflexive if $\sigma_{M}$ (resp. $\left.\tau_{N}\right)$ is an isomorphism.

Lemma 2.9. Let $P$ an $X \times X^{\prime}$-graded $R-R^{\prime}$-bimodule. The following are equivalent:

(a) $\lambda^{P}: \hat{R} \rightarrow H\left(P_{R^{\prime}}, P_{R^{\prime}}\right)$ (resp. $\chi^{P}: \hat{R}^{\prime} \rightarrow H\left({ }_{R} P,{ }_{R} P\right)$ is an) is an isomorphism.

(b) For every $x \in X$ (resp. $\left.x^{\prime} \in X^{\prime}\right), u_{R(x)}^{P}$ (resp. $c_{R^{\prime}\left(x^{\prime}\right)}^{P}$ ) is an isomorphism.

(c) For every $x \in X$ (resp. $\left.x^{\prime} \in X^{\prime}\right),(x) R$ (resp. $R^{\prime}\left(x^{\prime}\right)$ ) is $P$ reflexive.

$P$ is said to be faithfully balanced if both $\lambda^{P}$ and $\chi^{P}$ are isomorphisms.

Theorem 2.10. Let $P$ be an $X \times X^{\prime}$-graded $R$ - $R^{\prime}$-bimodule. The following conditions are equivalent:

(a) $\left(H\left(-,{ }_{R} P\right), H\left(-, P_{R^{\prime}}\right)\right)$ define a Morita duality between $(G, X, R)-g r$ and $g r-\left(R^{\prime}, X^{\prime}, G^{\prime}\right)$.

(b) For every $x \in X$ and $x^{\prime} \in X^{\prime}$, every graded quotient of $(x) R, R^{\prime}\left(x^{\prime}\right),{ }_{x} P$ and $P_{x^{\prime}}$ is $P$-reflexive.

(c) $P$ is graded faithfully balanced, $\left\{P_{x^{\prime}} \mid x^{\prime} \in X^{\prime}\right\}$ is a set of injective cogenerators in the category $(G, X, R)-g r$ and $\left\{{ }_{x} P \mid x \in X\right\}$ is a set of injective cogenerators in the category $g r-\left(R^{\prime}, X^{\prime}, G^{\prime}\right)$.

\section{Duality Theorems}

The main goal of this section is to extend the Duality Theorems of Cohen and Montgomery $[\mathrm{CM}]$ to our setting. There are other alternative extensions which can be found in $[\mathrm{Q}],[\mathrm{B} 1],[\mathrm{B} 2]$ and $[\mathrm{AN}]$ (see also $[\mathrm{A}]$ ). 
The originality of our methods is that they are purely categorical. We give some equivalences of categories which will be useful in applications.

Let $R$ be a $G$-graded ring and $X$ a left $G$-set. $X$ will be considered as a right $G$-set by $x g=g^{-1} x(x \in X, g \in G)$.

Let $P=\underset{x \in X}{\ominus} R(x)$. For cvery $x \in X$ let $u_{x}: R(x) \rightarrow P$ be the canonical monomorphism and $\pi_{x}: P \rightarrow R(x)$ the canonical projection. $P$ becomes an $X \times X$-graded $R$ - $R$-bimodule by

$$
r u_{x}(a) s=u_{g x}(r a s) \quad\left(r \in R_{g}, a, s \in R\right)
$$

and the $X \times X$-grading

$$
P_{(x, y)}=u_{x}\left(R(x)_{y}\right) \quad(x, y \in X) .
$$

Moreover ${ }_{R} P$ and $P_{R}$ are faithful modules.

Note that $P$ is the $X \times X$-graded $R$-R-bimodule associated to the identity functor in $g r-(R, X, G)$. (See Definition 1.4).

Given $x, y \in X$ we write $R_{x y^{-1}}=\sum_{x=g y} R_{g}$. Also if $r \in R$, then $r_{x y^{-1}}$ will denote $\sum_{x=g y} r_{g}$. Note that $R_{x y^{-1}}$ (resp. $r_{x y^{-1}}$ ) is both the component of degree $x$ of $(y) R$ (resp. of $r$ considered in $(y) R$ ) and the component of degree $y$ of $R(x)$ (resp. of $r$ in $R(x)$ ).

Remark. Let $F C M_{X}(R)$ be the ring of all finite column square $X$ matrices with entries in $R$. Since ${ }_{R} P$ is free and $\left\{u_{x}(1) \mid x \in X\right\}$ is a basis of ${ }_{R} P$, then the map

$$
\begin{aligned}
\Phi: \operatorname{End}\left({ }_{R} P\right) & \longrightarrow F C M_{X}(R) \\
f & \mapsto M_{f}
\end{aligned}
$$

(bcing $M_{f}$ the square $X$-matrix having $\pi_{y} f u_{x}(1)$ at the $(x, y)-t h$ entry) is a ring isomorphism. Moreover, it is quite easy to see that $\Phi\left(\operatorname{End}_{g r-(R, X, G)}(P)\right)=\left\{a \in F C M_{X}(R) \mid\right.$ for every $x, y \in X$, the $(x, y)-t h$ entry belongs to $\left.R_{x: y^{-1}}\right\}$. Thus $\Phi$ gives a matricial description of $\left(\right.$ End $_{g r-(R, X, G)}(P)$. This matricial description has been obtained by $\mathrm{T}$. Albu [A] for the particular case $X=G$.

Let $S=\operatorname{End}_{g r-\langle R, X, G)}(P)$. If we consider $S$ trivially graded by $G^{\prime}=$ $\{e\}$ and $X^{\prime}=G^{\prime}$ as a left $G^{\prime}$-set, then $P$ is naturally an $X^{\prime} \times X$-graded $S$-R-bimodule.

Since $P$ is an $X \times X$-graded $R$ - $R$-bimodule, then then map $r \mapsto \Lambda_{r}$ which associates to an element $r \in R$ the left multiplication-by-r map in $P$ is a ring homomorphism $\Lambda: R \rightarrow S$. Since ${ }_{R} P$ is faithful, $\Lambda$ is injective. Note that for every $r \in R_{x y^{-1}}, \pi_{y} \Lambda_{r} u_{x}=\lambda_{r}: R(x) \rightarrow R(y)$.

Let $I=H\left({ }_{R} P, P\right)$. Clearly $I=\left\{\alpha \in S \mid \alpha u u_{x}=0\right.$ for almost all $\left.x \in X\right\}$. 
Lemma 3.1. $I$ is an $S$-R-bisubmodule of $S$ and it is isomorphic to ${ }_{S} P_{R}$.

Proof: Let $\varphi: P \rightarrow S$ be the additive homomorphism given by

$$
\varphi\left(u_{x}(r)\right) u_{y}=u_{x} \lambda_{r_{x y-1}} \quad(r \in R, x, y \in X) .
$$

Note that for every $x, y \in X, u_{x} \lambda_{r_{x y-1}}=\Lambda_{r_{x y-1}} u_{y}$.

Let $\alpha \in S, r \in R$ and $x, y, z \in X$. Then $\pi_{y} \varphi\left(\alpha u_{x}(r)\right) u_{z}=$ $\pi_{y}\left(\sum_{w \in X} \varphi u_{w} \pi_{z} \alpha u_{x}(r)\right) u_{z}=\pi_{y} \varphi\left(u_{y} \pi_{y} \alpha u_{x}(r)\right) u_{z}=\lambda_{\left(\pi_{y} \alpha u_{x}(r)\right)_{y z}-1}$. If $g \in G$, then $r_{g} \in R(x)_{x g}$, therefore $\pi_{y} \alpha u_{x}\left(r_{g}\right) \in R(y)_{x g}$. Thus $\left(\pi_{y} \alpha u_{x}(r)\right)_{y z^{-1}}=\sum_{x g=z} \pi_{y} \alpha u_{x}\left(r_{g}\right)=\pi_{y} \alpha u_{x}\left(r_{x z^{-1}}\right)$. Then $\pi_{y} \varphi\left(\alpha u_{x}(r)\right) u_{z}=$ $\lambda_{\pi_{y} \alpha u_{x}\left(r_{x z-1}\right)}=\pi_{y} \alpha u_{x} \lambda_{r_{x z-1}}=\pi_{y} \alpha \varphi\left(u_{x}(r)\right) u_{z}$. Wo conclude that for every $x \in X, r \in R$ and $\alpha \in S, \varphi\left(\alpha u_{x}(r)\right)=\alpha \varphi\left(u_{x}(r)\right)$ and hence $\varphi$ is $S$-linear.

Let now $r, s \in R, x, y \in X$. Then $\varphi\left(u_{x}(r) s\right) u_{y}=\varphi\left(u_{x}(r s)\right) u_{y}=$ $u_{x} \lambda_{(r s)_{r: y^{-1}}}$. But $(r s)_{x y^{-1}}=\sum_{w \in X} r_{r x w p^{-1}} s_{w x^{-1}}$. Thus $\varphi\left(u_{x}(r) s\right) u_{y}=$ $\sum_{w \in X} u_{x} \lambda_{r_{x w^{-1}}} \lambda_{s_{w y-1}}=\sum_{w \in X} \varphi\left(u_{x}(r)\right) u_{w} \lambda_{s_{w y-1}}=\varphi\left(u_{x}(r)\right) \sum_{w \in X} u_{w} \lambda_{s_{w y-1}}=$ $\varphi\left(u_{x}(r)\right) \sum_{w \in X} \Lambda_{s_{w y-1}} u_{y}=\varphi\left(u_{x}(r)\right) \Lambda_{s} u_{y}$. Therefore $\varphi\left(u_{x}(r) s\right)=\varphi\left(u_{x}(r)\right) s$, that is, $\varphi$ is an homomorphism of right $R$-modules.

Let $p=\sum_{x \in X} u_{x}\left(r_{x}\right)$ be an element of $\operatorname{Ker}(\varphi)$. Then for all $x, y \in X, 0=$ $\pi_{x} \varphi(p) u_{y}=u_{x} \lambda_{\left(r_{x}\right)_{x y^{-1}}}$. Thus $r_{x}=\sum_{y \in X}\left(r_{x}\right)_{x y^{-1}}=0$. We conclude that $\varphi$ is injective.

Let $r \in R$ and $x \in X$. Then for every $y \in X, \varphi\left(u_{x}(r)\right) u_{y}=0$ unless $x \in \operatorname{Supp}(r) y$. Therefore $\varphi(P) \subseteq I$.

Finally, let $\alpha \in I$. For every $x \in X$ let $r_{x}=\sum_{y \in X} \pi_{x} \alpha u_{y}(1)$. Since $u_{y}(1) \in R(y)_{y}, \pi_{x} \alpha u_{y}(1) \in R(x)_{y}$ and hence $\left(r_{x}\right)_{x y^{-1}}=\pi_{x} \alpha u_{y}(1)$. Let $p=\sum_{x \in X} u_{x}\left(r_{x}\right)$. Then, for every $y \in Y, \varphi(p) u_{y}\left(r^{\prime}\right)=\sum_{x \subset X} u_{x}\left(\left(r_{x}\right)_{x y-1} r^{\prime}\right)=$ $\sum_{x \in X} u_{x}\left(\pi_{x} \alpha u_{y}(1) r^{\prime}\right)=\alpha u_{y}\left(r^{\prime}\right)$. Thus $\varphi(p)=\alpha$. We conclude that $\varphi(P)=I$.

For every subset $F$ of $X$ let $p_{F} \in S$ given by $p_{F}(m)=\sum_{x \in F} u_{x} \pi_{x}(m)$. Plainly $p_{F} p_{F^{\prime}}=p_{F \cap F^{\prime}}$, for every two subset $F$ and $F^{\prime}$ of $X$. Therefore, $p_{F}$ is an idempotent for every subset $F$ of $X$. Moreover $p_{F}+p_{X-F}=1$ for every $F \subseteq X$. 
For every $x \in X$ let $p_{x}=p_{\{x\}}\left(=u_{x} \pi_{x}\right) . \quad\left\{p_{x} \mid x \in X\right\}$ is a set of orthogonal idempotents of $S$. For every subset $F$ of $X, P_{F} \in I$ if and only if $F$ is finite.

Lemma 3.2. (a) Let $\alpha \in S, \alpha \in I$ if and only if there exists a finite subset $F$ of $X$ such that $\alpha=\alpha p_{F}$.

(b) I is idempotent.

(c) $I=\underset{x \in X}{\oplus} S p_{x}$ and $S p_{x}=R p_{x}$ for every $x \in X$.

Proof: Let $\alpha \in I$. There exists a finitc subset $F$ os $X$ such that $\alpha u_{x}=0$ for all $x \in X-F$. Then $\alpha=\alpha p_{F}$. This proves (a) and (b).

The sum $\sum_{x \in X} S p_{x}$ is direct because $\left\{p_{x} \mid x \in X\right\}$ is a set of orthogonal idempotents. Moreover, for every $x \in X$ and $\alpha \in S, \alpha p_{x}=\Lambda_{r} p_{x}$ where $r=\sum_{y \in X} \pi_{y} \alpha u_{x}(1)$. Indeed, let $m \in P$, then $\alpha p_{x}(m)=\alpha u_{x} \pi_{x}(m)=$ $\sum_{y \in X} u_{y} \pi_{y}\left(\alpha u_{x}(1)\right) \pi_{x}(m)=\sum_{y \in X} u_{y}\left(r_{y x^{-1}}\right) \pi_{x}(m)=\sum_{y \in X} u_{y} \lambda_{r_{y x}-1}\left(\pi_{x}(m)\right)=$ $\sum_{y \in X} \Lambda_{r y x^{-1}} u_{x} \pi_{x}(m)=\Lambda_{r} p_{x}(m)$.

Given a left $A$-module $M$, then $\operatorname{Gen}\left({ }_{A} M\right)$ denotes the full subcategory of $A$-mod formed by the right $A$-modules generated by $A M$. Next Lemma is obvious.

Lemma 3.3. Let $I$ be an idempotent left ideal of a ring $A$. Then $\operatorname{Gen}\left({ }_{A} I\right)=\{M \in A-\bmod \mid I M=M\}$.

Theorem 3.4. The pair of adjoint functors $(G, X, R)-g r \underset{H\left({ }^{2} P_{1}-\right)}{P \hat{\otimes}_{R-}}$ $S$-mod, defines an equivalence of categories between $(G, X, R)-g r$ and $\operatorname{Gen}(S I)$.

Proof: Let $T=P \hat{\otimes}_{R^{-}}$and $H=H(S P,-)$. Let $u$ be the unit of the adjunction pair $(T, H)$ and $c$ the counit. Let us remark that $u$ and $c$ are given by:

$$
\begin{array}{ll}
u_{M}: M \longrightarrow H T(M) & (M \in(G, X, R)-g r) \\
{\left[u_{M}(m)\right](p)=\sum_{x \in X} m_{x} \otimes_{x} p} & (m \in M, p \in P) \\
c_{N}: T H(N) \longrightarrow N & (N \in \bmod -S) \\
c_{N}(f \otimes p)=f(p) & \left(x \in X, p \in{ }_{x} P, f \in H\left(P_{S}, N\right)_{x}\right.
\end{array}
$$

For every $M \in(G, X, R)-g^{r}, u_{M}$ is nothing but the composition of the following canonical isomorphisms: $M \cong \underset{x \in X}{\ominus} P(x) \hat{\otimes}_{R} \dot{M}$ (see Lemma 
1.1) $=I P \hat{\otimes}_{R} M \cong \operatorname{Hom}\left(S I, P \hat{\otimes}_{R} M\right)=H T(M)$. Therefore $u_{M}$ is an isomorphism.

Let now $N \in S$-mod. Let $\varphi_{N}: T H(N) \rightarrow I N$ be the composition of the following canonical isomorphisms: $\varphi_{N}: P \hat{\otimes}_{R} \operatorname{Hom}\left({ }_{S} I, N\right) \cong$ $\bigoplus_{x \in X} R(x) \hat{\otimes}_{R} \operatorname{Hom}\left({ }_{S} I, N\right) \cong \operatorname{Hom}\left({ }_{S} I, N\right) \cong I N$. Then the following diagram is commutative:

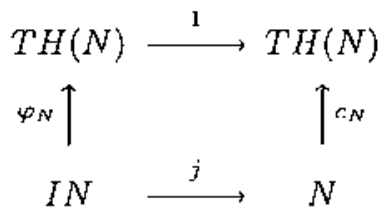

where $j$ is the inclusion map.

Thus $c_{N}$ is an isomorphism if and only if $N=I N$. Now Lemma 3.3 applies.

Remark. Theorem 3.4 implies [CM, Theorem 2.2] and [NRV, Theorem 2.13].

Recall that a ring $A$ (not necessarily with unit) is said to have local units if for every finite subset $F$ of $A$ there exists an idempotent $e$ of $A$ such that $F \in e A e$. We will say that a left ideal $I$ of $S$ has local units if it has local units when it is considered as a ring without unit.

Lemma 3.5. Let $A$ be a ring and I a left ideal of $A$ which has local units. Let $B$ a subring of $A$ which contains 1 . The restriction of scalor functor induces an equivalence between $\operatorname{Gen}\left({ }_{A} I\right)$ and $\operatorname{Gen}\left({ }_{B} I\right)$.

Proof: Let $F: A-\bmod \rightarrow B$-mod be the restriction of scalars functor. $F$ is obviously faithful. Let $M, N \in \operatorname{Gen}\left({ }_{A} I\right)$ and $f \in \operatorname{Hom}_{B}(M, N)$. Let $m \in M$ and $a \in A$. Iset $e$ be an idempotent in $I$ such that $e m=m$. Then $f(a m)=f(a e m)=a e f(e m)=a f(m)$. Therefore $f \in \operatorname{Hom}_{A}(M, N)$ and hence $F$ is full.

Let now $M \in \operatorname{Gen}\left({ }_{B} I\right)$. For every $m \in M$ and $a \in A$ let $a m=(a e) m$ where $e$ is an idempotent in $I$ such that $m=e m$. This definition does not depend on the choice of $e$ because if $e m=m=e^{\prime} m$ there exists $e^{\prime \prime}$ idempotent in $I$ such that $e, e^{\prime} \in e^{\prime \prime} A e^{\prime \prime}$. Thus (ae)m $=\left(a e^{\prime \prime}\right) e m=$ $\left(a e^{\prime \prime}\right) m=\left(a e^{\prime \prime}\right) e^{\prime} m=\left(a e^{\prime}\right) m$. This gives a structure of left $A$-module on $M$ and $F(M)=M$. 
Corollary 3.6. For every subring $A$ of $S$ which contains $I, P \hat{\otimes}_{R}-$; $(G, X, R)-g r \rightarrow A-\bmod$ defines an equivalence of categories between $(G, X, R)-g r$ and $\operatorname{Gen}\left({ }_{A} I\right)$.

Remark. Last corollary generalizes [A, Theorem 2.5].

Let $G, X, R, P$ and $S$ as above. Let $H$ be a group and assume that $X$ has an structure of $G$ - $H$-set. Let $\overline{\operatorname{END}}\left(P_{R}\right)_{H}=\left\{\alpha \in \operatorname{End}\left(P_{R}\right) \mid \alpha\left(P_{x}\right) \subseteq\right.$ $P_{x H}$ for every $\left.x \in X\right\} . \overline{\operatorname{END}}\left(P_{R}\right)$ is a subring of $\operatorname{End}\left(P_{R}\right)$.

For every $h \in H$ let $\operatorname{END}\left(P_{R}\right)_{h}=\left\{\alpha \in \operatorname{End}\left(P_{R}\right) \mid \alpha\left(P_{x}\right) \subseteq P_{x h^{-3}}\right.$ for every $x \in X\}$. For every $h, h^{\prime} \in H, \operatorname{END}\left({ }_{R} P\right)_{h} \cdot \operatorname{END}\left({ }_{R} P\right)_{h^{\prime}} \subseteq$ $\operatorname{END}\left({ }_{R} P\right)_{h h^{\prime}}$. Therefore $\operatorname{END}\left({ }_{R} P\right)_{H}=\sum_{h \in H} \operatorname{END}\left({ }_{R} P\right)_{h}$ is a subring of $\overline{\operatorname{END}}\left({ }_{R} P\right)_{H}$.

Moreover for every $h \in H$ the homomorphism $\bar{h}: P \rightarrow P$ given by $\bar{h} u_{x}=u_{x h^{-1}}(x \in X)$ belongs to $\operatorname{END}\left(P_{R}\right)_{h}$ and $\bar{h} \bar{h}^{\prime}=\overline{h h^{\prime}}$ for every $h, h^{\prime} \in H$. Therefore $\operatorname{END}\left({ }_{R} P\right)_{H}$ is an $H$-Clifford system.

Lemma 3.7. The sum $\sum_{h \in H} \operatorname{END}\left({ }_{R} P\right)_{h}$ is direct if and only if $H$ acts freely on $X$. In this case $\operatorname{END}\left({ }_{R} P\right)$ is a skew group ring over $H$ with underground ring $S$.

Proof: Let $h \in H$ and $0 \neq \alpha \in \operatorname{END}\left({ }_{R} P\right)_{h} \cap \sum_{h^{\prime} \neq h} \operatorname{END}\left({ }_{R} P\right)_{h^{\prime}}$. Let $x \in X$ such that $\alpha u_{x} \neq 0$. Then $\alpha u_{x}(1) \neq 0$. Let $y \in X$ such that $\pi_{y} \alpha u_{x}(1) \neq 0$. Since $u_{x}(1) \in P_{x}$, then $\pi_{y} \alpha u_{x}(1) \in R(y)_{x h^{-1}}$. Let $g \in \operatorname{Supp}\left(\pi_{y} \alpha u_{x}(1)\right)$. Then $x h^{-1}=g^{-1} y$. Let $h_{1}, h_{2}, \ldots, h_{n} \in H-\{h\}$, and $\alpha_{1} \in \operatorname{END}\left({ }_{R} P\right)_{h_{1}}, \alpha_{2} \in \operatorname{END}\left({ }_{R} P\right)_{h_{2}}, \ldots, \alpha_{n} \in \operatorname{END}\left({ }_{k} P\right)_{h_{n}}$ : such that $\alpha=\sum_{i=1}^{n} \alpha_{i}$. Let $i=1,2, \ldots, n$ such that $\alpha_{i} u_{x}(1) \neq 0$. By repeating the same argument, one has that $x h_{i}^{-1}=g^{-1} y=x h^{-1}$. Thus $X$ is not a free left $H$-set.

Conversely, assume that the sum $\sum_{h \in H} \operatorname{END}\left({ }_{R} P\right)_{h}$ is direct. Let $x \in X$ and $h \in H$ such that $x h=x$. Then $0 \neq P_{x} \in \operatorname{END}\left({ }_{R} P\right)_{e} \cap \operatorname{END}\left(P_{R}\right)_{h-1}$ and hence $h=e$.

Let $X$ be an $G$ - $H$-set. Consider the following relation of equivalence on $X$ :

$$
x \sim y \Longleftrightarrow y \in x H
$$

Then $X / H=\{x H \mid x \in X\}$ is the set of equivalent classes of $X$ by the relation of equivalence $\sim$. $G$ acts on $X / H$ by $g(x H)=g x H$. The map $\xi: x \mapsto x H$ is a homomorphism of right $G$-set. 
Let $X$ be a set and $I$ an ideal. By $F M_{X}(I)$ we will denote the set of all square $X$-matrices over $I$ having almost all entries equal to zero.

Theorem 3.8. Let $H$ and $G$ be two groups, $X$ an $G$ - $H$-set and $R$ a G-graded ring. Let $P=\underset{x \in G}{\oplus} R(x)$ and $U=\underset{C \in X / H}{\oplus} R(C)$. Let $A=$ $\overline{\operatorname{END}}\left(P_{R}\right)_{H}, S=\operatorname{End}_{g r-(R, X / H, G)}(U), J=\{\alpha \in A \mid \alpha(R(x))=0$ for almost all $x \in X\}$ and $I=\{\alpha \in S \mid \alpha(R(C))=0$ for almost all $C \in$ $X / H\}$.

There exists an isomorphism $\Phi: A \rightarrow F C M_{H}(S)$ such that $\Phi(J)=$ $F M_{H}(I)$.

Proof: Let $\xi: X \rightarrow X / H$ be the map which associates to $x \in X$ the class $x H$. Set $Q=T_{\xi}(P)$.

Plainly $Q=\underset{x \in X}{\oplus} R(x H)=U^{(H)}$. Therefore there is a canonical isomorphism $\Phi: \operatorname{End}_{(G, X / H, R)-g r}(Q) \rightarrow F C M_{H}\left(\operatorname{End}_{(G, X / H, R)-g r}(U)\right)$ such that $\Phi(K)=F M(I)$ being $K=\left\{\alpha \in\right.$ End $_{(G, X / H, R)-g r}(Q) \mid u_{x} \alpha=$ 0 for almost all $x \in X\}$. The theorem follows by realizing that $\operatorname{End}_{(G, X / H, R)-g_{r}}(Q)=\overline{\operatorname{END}}\left({ }_{R} P\right)_{H}$ and hence $J=K$.

Remark. Last theorem implies [CM, Theorem 3.5], [B2, Theorem 2.2] and [NRV, Corollary 2.2].

Lemma 3.9. Let $A$ be a ring and $X$ a set. Let $B=M_{X}(A)$ be the finite row and column matrix ring over $R$ indexed by $X$. Let $x \in X$ and $P_{x}$ the $X \times X$ matrix having 1 at the $(x, x)$-th entry and zeros elsewhere. The functor $\operatorname{Hom}\left({ }_{B} B p_{x},-\right)$ defines an equivalence of categories between $\operatorname{Gen}\left({ }_{B} B p_{x}\right)$ and $A$-mod.

Proof: Let $P=B p_{x} .{ }_{B} P$ is finitely generated and projective and End $\left({ }_{B} P\right)$ is isomorphic to $A$. It only remains to prove that ${ }_{B} P$ gencrates each of its submodules and apply [F, Theorem 2.6 and Lemma 2.2].

Let $M$ be a submodule of ${ }_{B} P$. For any $y \in X$ let $M_{y}=\{\alpha(y, x) \mid \alpha \in$ $M\}$. If $y, z \in X$ and $\alpha \in M$, then $\left(e_{z, y} \alpha\right)(z, x)=\alpha(y, x), e_{z, y}$ being the $X \times X$-matrix having 1 at the $(z, y)$-entry and zeros elsewhere. Therefore, $M_{y}=M_{z}$, that is, $N=M_{y}$ does not depend on $y \in X$.

Let $M^{\prime}=\{\alpha \in P \mid \alpha(y, x) \in N$ for all $y \in X\}$. We claim that $M=M^{\prime}$. The inclusion $M \subseteq M^{\prime}$ is clear. Let $\alpha \in M^{\prime}$. For every $y \in X$ there exists $\beta_{y} \in M$ such that $\alpha(y, x)=\beta_{y}(y, x)$. Then $\alpha=\sum_{\alpha(y, x) \neq 0} p_{y} \beta_{y} \in M$.

For every $a \in N$ let $a$ be the $X \times X$ matrix having $a$ at the $(x, x)-t h$ entry and zeros elsewhere. Let $f_{a}: P \rightarrow M$ be the right multiplication 
by $\hat{a}$ map. Then, for every $\alpha \in M, \alpha=\sum_{\alpha(y, x) \neq 0} f_{\alpha(y, x)}\left(e_{(y, x)}\right)$. Therefore $M$ is generated by ${ }_{i} P$.

Corollary 3.10. With the same hypothesis and notation than Theorem 2.8, the categories $\operatorname{Gen}\left({ }_{A} J\right)$ and $\operatorname{Gen}\left({ }_{S} I\right)$ are equivalent.

Proof: Let $B=F C M_{H}(S)$ and $C=M_{H}(S)$. Let $P=C p_{e}$ being $p_{e}$ the $H \times H$-matrix having $1 \in S$ at the $(e, e)$-position and zcros elsewhere.

By Lernma $3.9, F=\operatorname{Hom}(C P,-): \operatorname{Gen}\left(C_{C} P\right) \rightarrow S$-mod is an equivalence of catcgories. Let $Q=F M_{X}(J) p_{e}$. Then $F(Q) \cong I$ and hence $F$ induces an equivalence of categories betwcen $\operatorname{Gen}(C Q)$ and $\operatorname{Gen}\left({ }_{S} I\right)$.

Let $K=F M_{H}(I) . \quad K$ is isomorphic to $Q^{(H)}$ as a left $C$-module. Therefore $\operatorname{Gen}(C K)=\operatorname{Gen}(c Q)$.

By Lemma $3.5 \mathrm{Gen}(C K)$ is equivalent to $\operatorname{Gen}\left({ }_{B} K\right)$.

Now, by using the isomorphism $\Phi$ of Theorem 3.8, we have that $\operatorname{Gen}\left({ }_{B} K\right)$ is equivalent to $\operatorname{Gen}\left({ }_{A} J\right)$.

We conclude that $\operatorname{Gen}\left({ }_{B} J\right)$ is equivalent to $\operatorname{Gen}\left(S_{S} I\right)$.

We finish this section with a version of Cohen-Montgomery Duality Theorem for actions.

Theorem 3.11. Let $R * G$ be a crossed product and $H$ a subgroup of $G$. Let $P=\underset{C \in G / H}{\ominus} R(C)$. Then $\operatorname{Fnd}_{g r-(R, G / H)}(P)$ is isomorphic to $F C M_{G / H}(R * H)$.

Proof: For every $g \in G$ there is a unit $\bar{g}$ in $R * G$ of degree $g$. Then, for every $g \in G$ and every $C \in G / H$, left multiplication by $\bar{g}$ is a graded isomorphism between $R(C)$ and $R(g C)$. Thereforc $P \cong$ $R(H)^{(G / H)}$ in $(G / H, R)-g r$ and hence End $_{g r-(R, G / H)}(P)$ is isomorphic to $F C M_{G / H}\left(\right.$ Fnd $_{g r-(R, G / H)}(R(H))$. Finally, End $_{g r-(R, G / H)}(R(H)) \cong$ $R * H$, by Lomma 1.5 .

\section{Separable functors}

Separable functors have been introduced in [NVV] and studies in [NVV] and [Ra]. Let us recall the definition.

Definition. Let $F: \mathcal{C} \rightarrow \mathcal{D}$ be a functor between two arbitrary categories $\mathcal{C}$ and $\mathcal{D} . \quad F$ is said to be separable if for any two objects $C, C^{\prime} \in \mathcal{C}$, there is a map $\varphi=\varphi_{C, C^{\prime}}: \operatorname{Hom}_{\mathcal{D}}\left(F C, F C^{\prime}\right) \rightarrow \operatorname{Hom}_{C}\left(C, C^{\prime}\right)$ such that:

SF1. For all $f \in \operatorname{Hom}_{C}\left(C, C^{\prime}\right), \varphi(F f)=f$. 
SF2. For every commutative diagram in $\mathcal{D}$ of the type

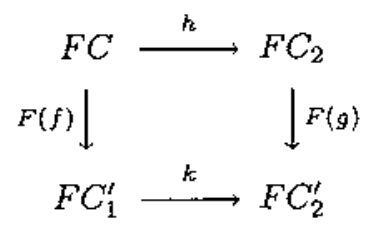

the following diagram in $\mathcal{C}$ is also commutative

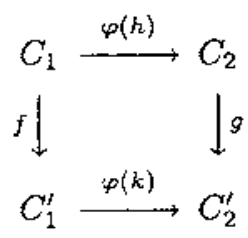

In this section we first recall a fundamental theorem of Rafael [Ra] on adjoint separable functors. Then we study conditions for the functors $T^{*}$ and $T_{*}$, defined in Section 1, to be separable. When $R=R^{\prime}$ and $\rho$ is the identity map on $R$, then $T^{*}$ is always separable while the separability 'test for $T_{*}$ can be simplified.

In [Ra] the authors give a complete characterization of separable adjoint functors in terms of the unit and counit of the adjunction. Explicitly they obtain the following result:

Theorem 4.1 [Ra]. Let $F: \mathcal{C} \rightarrow \mathcal{D}$ and $G: \mathcal{D} \rightarrow \mathcal{C}$ be functors such that $F$ is left adjoint of $G$. Let $u: 1_{\mathcal{C}} \rightarrow G F$ be the unit and $c: F G \rightarrow 1_{\mathcal{D}}$ the counit of the adjunction.

(a) $F$ is separable if and only if $u$ splits (i.e. There exists a natural transformation $\varepsilon: G F \rightarrow 1_{C}$ such that $\varepsilon_{C} \circ u_{C}=1_{C}$ for every $C \in \mathcal{C})$.

(b) $G$ is separable if and only if $c$ cosplits (i.e. There exists a natural tronsformation $\mu: 1_{D} \rightarrow F G$ such that $c_{D} \circ \mu_{D}=1_{D}$ for every $D \in \mathcal{D})$.

For the sake of completeness we give a proof different from the one given in [Ra].

Proof: By duality it is enough to prove (a).

Assume that $F$ is separable. For every $C, C^{t} \in \mathcal{C}$ let

$$
\varphi=\varphi_{C, C^{\prime}}: \operatorname{Hom}_{\mathcal{D}}\left(F C, F C^{\prime}\right) \rightarrow \operatorname{Hom}_{\mathcal{C}}\left(C, C^{\prime}\right)
$$

be a map satisfying SF1 and SF2. 
For every $C \in \mathcal{C}$, set $\varepsilon_{C}=\varphi\left(c_{F C}\right) \in \operatorname{Hom}_{C}(G F(C), C)$.

First we prove that $\varepsilon: G F \rightarrow 1_{\mathcal{C}}$ is a natural transformation. Indeed, let $f \in \operatorname{Hom}_{\mathcal{C}}\left(C, C^{\prime}\right)$, then the diagram

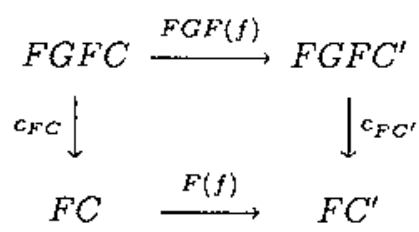

is commutative, because $c$ is a natural transformation. Therefore the following diagram is also commutative:

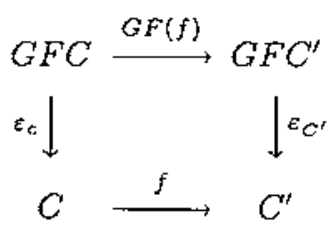

Now we prove that $\varepsilon_{C} u_{C}=1_{C}$ for every $C \in \mathcal{C}$. Indeed, the well known formula $c_{F C} \circ F\left(u_{C}\right)=1_{F C}$ implies the commutativity of the following diagram

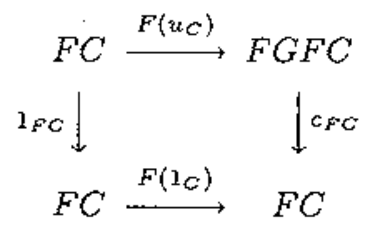

and SF2 implies the commutativity of the following diagram:

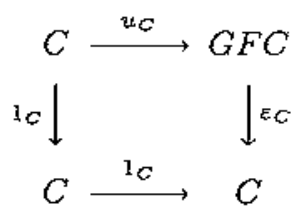

Conversely, let $\varepsilon: G F \rightarrow 1_{\mathcal{C}}$ be a natural transformation satisfying $\varepsilon \circ u=1$. For any $C, C^{\prime} \in \mathcal{C}$ let $\varphi: \operatorname{Hom}_{\mathcal{D}}\left(F C, F C^{\prime}\right) \rightarrow \operatorname{Hom}_{\mathcal{C}}\left(C, C^{\prime}\right)$ given by $\varphi(f)=\varepsilon_{C^{\prime}} \circ G(f) \circ u_{C}$.

If $f \in \operatorname{Hom}_{\mathcal{C}}\left(C, C^{\prime}\right)$, then $\varphi(F f)=\varepsilon_{C^{\prime}} G F(f) u_{C}=\varepsilon_{C^{\prime}} u_{C} f=f$.

On the other hand, if the following diagram in $\mathcal{D}$ is commutative

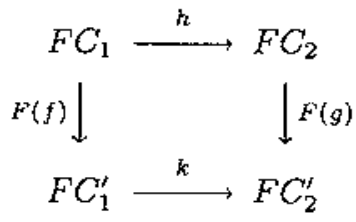


then the diagram

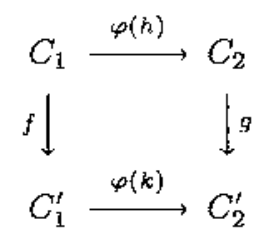

is also commutative because $g \circ \varphi(h)=g \circ \varepsilon_{C_{2}} \circ G(h) \circ u_{C_{1}}=\varepsilon_{C_{2}^{\prime}} \circ$ $G F(g) \circ G(h) \circ u_{C_{1}}=\varepsilon_{C_{2}^{\prime}} \circ G(F(g) \circ h) \circ u_{C_{1}}=\varepsilon_{C_{2}^{\prime}} \circ G(k) \circ G F(f) \circ u_{C_{1}}=$ $\varepsilon_{C_{2}^{\prime}} \circ G(k) \circ u_{C_{1}^{\prime}} \circ f=\varphi(k) \circ f$.

Theorem 4.1 can be used to give a simple criterion on when a right adjoint functor of the form $G: g r-(R, X, G) \rightarrow \mathcal{D}$ is separable, for $\mathcal{D}$ an additive category. Next proposition was inspired from Proposition 2.2 in [Ra].

Proposition 4.2. Let $\mathcal{C}$ be a full subcategory of $\mathrm{gr}-(R, X, G)$ such that $R(x) \in \mathcal{C}$ for every $x \in X$. Let $G: \mathcal{C} \rightarrow \mathcal{D}$ be a right adjoint functor, $F$ a left adjoint of $G$ and $c: F G \rightarrow 1_{C}$ the counit of the adjonction. The following conditions are equivalent:

(a) $G$ is separable.

(b) For any $x \in X$ there exists $m_{x} \in F G(R(x))_{x}$ satisfying the following two conditions:

(1) For any $x \in X, c_{R(x)}\left(m_{x}\right)=1$.

(2) For any $x \in X, g \in G$ and $r \in R_{g}, F G\left(\lambda_{r}\right)\left(m_{x g}\right)=$ $m_{x} r$ (where $\lambda_{r} \in \operatorname{Hom}_{g r-(R, X, G)}(R(x g), R(x))$ is the left multiplication-by-r map).

Proof: Assume first that $G$ is separable. By Theorem 4.1, there exists a natural transformation $\mu: 1_{\mathcal{C}} \rightarrow F G$ such that $c o \mu=1$. By Lemma 1.5 for every $x \in X$ there exists $m_{x} \in F G(R(x))$ such that $\mu_{R(x)}=\lambda_{m_{x}}$. Then $c_{R(x)}\left(m_{x}\right)=\left(c_{R(x)} \circ \mu_{R(x)}\right)(1)=1$. On the other hand, if $r \in R_{g}$, then for every $x \in X$ the following diagram

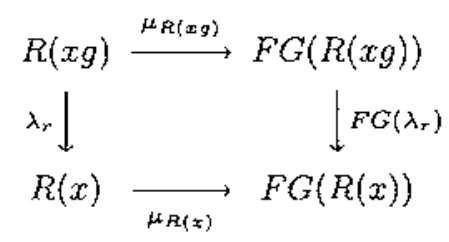

is commutative. Therefore $F G\left(\lambda_{r}\right)\left(m_{x g}\right)=\left[F G\left(\lambda_{r}\right) \circ \mu_{R(x ; g)}\right](1)=$ $\left[\mu_{R(x)} \circ \lambda_{r}\right](1)=m_{x} r$. 
Let now $\left.\left\{m_{x} \in F G(x)\right)_{x} \mid x \in X\right\}$ be a system of elements satisfying conditions (1) and (2). For every $N \in g r-(R, X, G)$ define $\mu_{N}: N \rightarrow$ $F G(N)$ by $\mu_{N}(n)=F G\left(\lambda_{n}\right)\left(m_{x}\right)$ if $n \in N_{x}$ and extend $\mu_{N}$ to $N$ by linearity.

$\mu_{M}$ is $R$-linear because if $n \in N_{x}$ and $r \in R_{g}$, then $\mu_{N}(n r)=$ $F G\left(\lambda_{n \tau}\right)\left(m_{x g}\right)=F G\left(\lambda_{n}\right) F G\left(\lambda_{r}\right)\left(m_{x g}\right)=F G\left(\lambda_{n}\right)\left(m_{x} r\right)=F G\left(\lambda_{n}\right)\left(m_{x}\right) r=$ $\mu_{N}(n) r$.

Now we prove that $\mu$ is a natural transformation. Let $f \in \operatorname{Hom}_{g r}-(R, X, G)$ $\left(N, N^{\prime}\right)$ and $n \in N_{x}$. Then

$$
\begin{aligned}
{\left[F G(f) \circ \mu_{N}\right](n)=F G\left(f \circ \lambda_{n}\right)\left(m_{x}\right)=} & F G\left(\lambda_{f(n)}\right)\left(m_{x}\right)= \\
& =\mu_{N^{\prime}}(f(n))=\left(\mu_{N^{\prime}} \circ f\right)(n) .
\end{aligned}
$$

Finally, if $N \in \mathcal{C}$ and $n \in N_{x}$, then $\left(c_{N} \circ \mu_{N}\right)(n)=\left[c_{N} \circ\right.$ $\left.F G\left(\lambda_{n}\right)\right]\left(m_{x}\right)=\left[\lambda_{n} \circ c_{R(x)}\right]\left(m_{x}\right)=\lambda_{n}(1)=n$.

Thus, applying Theorem 4.1 we conclude that $G$ is separable.

For the rest of this section we consider the following situation: Let $T=(\rho, \xi, \gamma)$ where $\rho: R \rightarrow R^{\prime}$ is a ring homomorphism, $\xi: X \rightarrow X^{\prime}$ is a map, $\gamma: G \rightarrow G^{\prime}$ is a group homomorphism, $R$ is a $G$-graded ring, $R^{\prime}$ is a $G^{\prime}$-graded ring, $X$ is a right $G$-set, $X^{\prime}$ is a right $G^{\prime}$-set and the following conditions hold:

$$
\rho\left(R_{g}\right)=R_{\gamma(g)}^{\prime} \quad \xi(x g)=\xi(x) \gamma(g) \quad(g \in G, x \in X) .
$$

Let $T^{*}$ and $T_{*}$ be the functors defined in Section 1 . We are going to study when $T^{*}$ and $T_{*}$ are scparable.

Proposition 4.3. Let $T=(\rho, \xi, \gamma)$ as above. The following conditions are equivalent:

(1) $T^{*}$ is separable.

(2) For every $x \in X$ there is $\varepsilon_{x} \in \operatorname{Hom}_{g r-\{R, X, G)}\left(T_{*}\left(R^{\prime}(\xi(x)), R(x)\right)\right.$ such that.

(a) For every $x \in X, \varepsilon_{x}\left(1^{x}\right)=1$.

(b) For every $x, y \in X, g \in G, r \in R_{g}$ and $r^{\prime} \in R^{\prime}(\xi(x g))_{\xi(y)}$,

$$
\varepsilon_{x}\left(\left(\rho(r) r^{\prime}\right)^{y}\right)=r \varepsilon_{x g}\left(\left(r^{\prime}\right)^{y}\right) \text {. }
$$

Proof: First let us note that the unit $u$ of the adjunction pair $\left(T^{*}, T_{*}\right)$ is given by:

$$
\begin{aligned}
& u_{M}: M \longrightarrow T_{*} T^{*}(M) \quad(M \in g r-(R, X, G)) \\
& m \mapsto \sum_{x \in X}\left(m_{x} \otimes 1\right)^{x}(m \in M) \text {. }
\end{aligned}
$$


On the other hand the canonical isomorphism $R^{\prime} \rightarrow R \otimes_{R} R^{\prime}$ induces an isomorphism $\phi_{x}: R^{\prime}(\xi(x)) \rightarrow T^{*}(R(x))$ for every $x \in X$.

(1) $\Rightarrow$ (2) Assume that $T^{*}$ is separable. By Theorem 4.1 there exists a natural transformation $\varepsilon: T_{*} T^{*} \rightarrow 1_{g r-(R, X, G)}$ such that $\varepsilon_{M} \circ u_{M}=1_{M}$ for every $M \in g r-(R, X, G)$.

For every $x \in X$ let $\varepsilon_{x}=\varepsilon_{R(x)} \circ T_{*}\left(\phi_{x}\right)$. We are going to see that $\left\{\varepsilon_{x} \mid x \in X\right\}$ satisfies conditions (a) and (b).

$$
\varepsilon_{x}\left(1^{x}\right)=\varepsilon_{R(x)}\left((1 \otimes 1)^{x}\right)=\left[\varepsilon_{R(x)} \circ u_{R(x)}\right](1)=1 .
$$

Let now $g, h \in G, r \in R_{g}$ and $r^{\prime} \in R^{\prime}(\xi(x g))_{\xi(y)}$. Let $\lambda_{r}: R \rightarrow R$ be the left multiplication by $r$ map and $\lambda_{\rho(r)}: R^{\prime} \rightarrow R^{\prime}$ left multiplication by $\rho(r)$. By Lemma 1.3 , one has

$$
\lambda_{r} \in \operatorname{Horm}_{g r-(R, X, G)}(R(x g), R(x))
$$

and

$$
\lambda_{\rho(r)} \in \operatorname{Hom}_{g^{r}-\left(R^{\prime}, X^{\prime}, G^{\prime}\right)}\left(R^{\prime}(\xi(x g)), R^{\prime}(\xi(x))\right) .
$$

Furthermore the following diagram is commutative

$$
\begin{aligned}
& T_{*}\left(R^{\prime}(\xi(x g))\right) \stackrel{T_{*}\left(\phi_{x g}\right)}{\longrightarrow} T_{*} T^{*}(R(x g)) \stackrel{\varepsilon_{R(x g)}}{\longrightarrow} R(x g)
\end{aligned}
$$

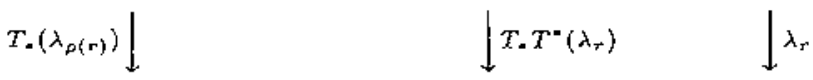

$$
\begin{aligned}
& T_{*}\left(R^{\prime}(\xi(x))\right) \stackrel{T_{*}\left(\phi_{x}\right)}{\longrightarrow} T_{*} T^{*}(R(x)) \stackrel{\epsilon_{R(x)}}{\longrightarrow} R(x)
\end{aligned}
$$

Therefore, $\varepsilon_{x}\left(\left(\rho(r) r^{\prime}\right)^{y}\right)=\left[\varepsilon_{R(x)} \circ T_{*}\left(\phi_{x}\right)\right]\left(\left(\lambda_{\rho(r)}\left(r^{\prime}\right)\right)^{y}\right)=\left[\varepsilon_{R(x)} \circ\right.$ $\left.T_{*}\left(\phi_{x}\right)\right]\left(\left[T_{*}\left(\lambda_{\rho(r)}\right)\right]\left(\left(r^{\prime}\right)^{y}\right)=\left[\lambda_{r} \circ \varepsilon_{x g}\right]\left(\left(r^{\prime}\right)^{y}\right)=r \varepsilon_{x g}\left(\left(r^{\prime}\right)^{y}\right)\right.$.

(2) $\Rightarrow$ (1) Assume that for every $x \in X$ therc exists $\varepsilon_{x} \in$ $\operatorname{Hom}_{(G, X, K)-g r}\left(T_{*}\left(R^{\prime}(\xi(x))\right), R(x)\right)$ satisfying conditions (a) and (b). For every $M \in g^{r}-(R, X, G)$ let $\varepsilon_{M}: T_{*} T^{*}(M) \rightarrow M$ be the homomorphism given by $\varepsilon_{M}\left(\left(m \otimes r^{\prime}\right)^{y}\right)=m \varepsilon_{x}\left(r^{\prime y}\right)$ whenever $m \in M_{x}$ and $r^{\prime} \in R^{\prime}(\xi(x))_{\xi(y)}$ and extended by linearity. Note that $\varepsilon_{M}$ is well defined. Indeed, let $m \in M_{x}, r \in R_{g}$ and $r^{\prime} \in R^{\prime}(\xi(x g))_{\xi(y)}$. Then $\varepsilon_{M}\left(m r \otimes r^{\prime}\right)=m r \varepsilon_{x \beta}\left(r^{\prime y}\right)=m \varepsilon_{x}\left(\left(\rho(r) r^{\prime}\right)^{y}\right)=\varepsilon_{M}\left(\left(m \otimes r r^{\prime}\right)^{y}\right)$.

It is an easy exercise to check that $\varepsilon_{M} \in \operatorname{Hom}_{g r-(R, X, G)}\left(T_{*} T^{*}(M), M\right)$. Moreover, for every $m \in M_{x},\left[\varepsilon_{M} \circ u_{M}\right](m)=\varepsilon_{M}\left((m \otimes 1)^{x}\right)=m \varepsilon_{x}\left(1^{x}\right)=$ $m$. It only remains to prove that $\varepsilon$ is a natural transformation from $T_{*} T^{*}$ to $1_{g r-(R, X, G)}$ and apply Theorem 4.1 .

Let $f \in \operatorname{Hom}_{g^{r-(R, X, G)}}(M, N), m \in M_{x}$ and $r^{\prime} \in R^{\prime}(\xi(x))_{\xi(y)}$. Then $\left[\varepsilon_{N} \circ T_{*} T^{*}(f)\right]\left(\left(m \otimes r^{\prime}\right)^{y}\right)=\varepsilon_{N}\left(\left(f(m) \otimes r^{\prime}\right)^{y}\right)=f(m) \varepsilon_{x}\left(r^{\prime y}\right)=$ $f\left(m \varepsilon_{x}\left(r^{\prime y}\right)\right)=f\left(\varepsilon_{M}\left(\left(m \otimes r^{\prime}\right)^{y}\right)\right)=\left[f \circ \varepsilon_{M}\right]\left(\left(m \otimes r^{\prime}\right)^{y}\right)$. 
Proposition 4.4. Let $T=(\rho, \xi, \gamma)$ as above. The following conditions are equivalent:

(a) $T_{*}$ is separable.

(2) There exists a system

$$
\begin{aligned}
& \sum=\left\{\left(a_{\left(x^{\prime}, i\right)}^{\prime}, b_{\left(x^{\prime}, i\right)}^{\prime}, x_{\left(x^{\prime}, i\right)}\right) \in R_{g_{\left(x^{\prime}, i\right)}^{\prime}}^{\prime} \times R_{\left(g_{\left(x^{\prime}, i\right)}^{\prime}\right)^{-1}}^{\prime} \times X \mid x^{\prime} \in X^{\prime},\right. \\
& \left.i=1,2, \ldots, m_{x^{\prime}}\right\}
\end{aligned}
$$

satisfying the following conditions.

(1) For every $x^{\prime} \in X^{\prime}$ and $i=1,2, \ldots, m_{x^{\prime}}, x^{\prime} g_{\left(x^{\prime}, i\right)}^{\prime}=\xi\left(x_{\left(x^{\prime}, i\right)}\right)$.

(2) For every $x^{\prime} \in X^{\prime}, \sum_{i=1}^{m_{x}} a_{\left(x^{\prime}, i\right)}^{\prime} b_{\left(x^{\prime}, i\right)}^{\prime}=1$.

(3) For every $r^{\prime} \in R_{g^{\prime}}^{\prime}$, and every $x^{\prime} \in X^{\prime}$, the following equality holds in $T_{*}\left(R^{\prime}\left(x^{\prime}\right)\right) \otimes_{R} R^{\prime}$.

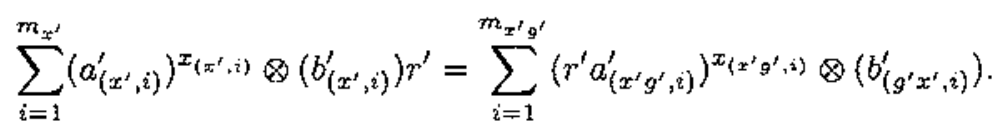

Proof: First let us remark that the counit $c: T^{*} T_{*} \rightarrow 1$ of the adjunction pair $\left(T^{*}, T_{*}\right)$ is given by:

$$
\begin{array}{cl}
c_{N}: T_{*}(N) \otimes R R^{\prime} \longrightarrow N & \left(N \in g r-\left(R^{\prime}, X^{\prime}, G^{\prime}\right)\right) \\
c_{N}\left(n^{x} \otimes r^{\prime}\right)=n r^{\prime} & \left(r^{\prime} \in R^{\prime}, n \in N_{\xi(x)}\right) .
\end{array}
$$

(a) $\Rightarrow$ (b) Assume that $T_{*}$ is separable. By Proposition 4.2 , for every $x^{\prime} \in X^{\prime}$ there exists $K_{x^{\prime}} \in\left(T_{*}\left(R^{\prime}\left(x^{\prime}\right)\right) \otimes_{R} R^{\prime}\right)_{x^{\prime}}$ such that

(1) $c_{R^{\prime}\left(x^{\prime}\right)}\left(K_{x^{\prime}}\right)=1$.

(2) For any $x^{\prime} \in X^{\prime}, g^{\prime} \in G^{\prime}$ and $r^{\prime} \in R_{g^{\prime}}^{\prime}, T^{*} T_{*}\left(\lambda_{r}\right)\left(K_{x^{\prime} g^{\prime}}\right)=K_{x^{\prime}} r$.

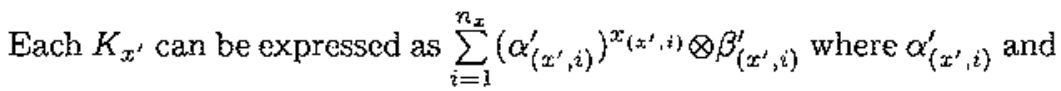
$\beta_{\left(x^{\prime}, 2\right)}^{\prime}$ are homogeneous for every possible $x^{\prime}$ and $i$. For every possible $x^{\prime}$ and $i$, let $g_{\left(x^{\prime}, i\right)}^{\prime}$ be the degree of $\alpha_{\left(x^{\prime}, i\right)}^{\prime}$ and $h_{\left(x^{\prime}, i\right)}^{\prime}$ the degree of $\beta_{\left(x^{\prime}, i\right)}^{\prime}$. Then $x^{\prime} g_{(x, i)}^{\prime}=\xi\left(x_{\left(x^{\prime}, i\right)}\right)$ and $\xi\left(x_{\left(x^{\prime}, i\right)}\right) h_{\left(x^{\prime}, i\right)}^{\prime}=x^{\prime}$.

Let $\sum=\left\{\left(a_{\left(x^{\prime}, i\right)}^{\prime}, b_{\left(x^{\prime}, i\right)}^{\prime}, x_{\left(x^{\prime}, i\right)}\right) \mid x^{\prime} \in X^{\prime}, i=1,2, \ldots, m_{x}\right\}$ be the system which results by eliminating in the system $\left\{\left(\alpha_{\left(x^{\prime}, i\right)}^{\prime}, \beta_{\left(x^{\prime}, i\right)}^{\prime}, x_{\left(x^{\prime}, i\right)}\right) \mid x^{\prime} \in\right.$ $\left.X^{\prime}, i=1,2, \ldots n_{x^{\prime}}\right\}$ any upla $\left\{\alpha_{\left(x^{\prime}, i\right)}^{\prime}, \beta_{\left(x^{\prime}, i\right)}^{\prime}, x_{\left(x^{\prime}, i\right)}\right)$ such that $g_{\left(x^{\prime}, i\right)}^{\prime} h_{(x, i)}^{\prime} \neq e$. Plainly $\sum$ satisfies (1). 
Since $1=c_{R^{\prime}\left(x^{\prime}\right)}\left(K_{x^{\prime}}\right)=\sum_{i=1}^{n_{x^{\prime}}} \alpha_{\left(x^{\prime}, i\right)}^{\prime} \beta_{\left(x^{\prime}, i\right)}^{\prime}$, then the homogeneous component of degree $e$ of this element is equal to 1 . But this homogeneous component is just $\sum_{i=1}^{m_{x^{\prime}}} a_{\left(x^{\prime}, i\right)}^{\prime} b_{\left(x^{\prime}, i\right)}^{\prime}$. Thus $\sum$ satisfies (2).

Consider $G^{\prime}$ as a right $G$-set by: $g^{\prime} g=g^{\prime} \gamma(g)\left(g \in G, g^{\prime} \in G^{\prime}\right)$. Consider in $M=T_{*}\left(R^{\prime}\left(x^{\prime}\right)\right)$ the following $G^{\prime}$-grading: A typical element $r^{\prime x}$ in $T_{*}\left(R^{\prime}\left(x^{\prime}\right)\right)_{x}$ has degree $g^{\prime} \in G^{\prime}$ if $r^{\prime} \in R_{g^{\prime}}^{\prime}$. Plainly $M \in g r-\left(R, G^{\prime}, G\right)$. Let $S=\left(\rho, I_{G^{\prime}}, \gamma\right)$. Then $S^{*}(M)=M \otimes_{R} R^{\prime} \in g r-\left(R^{\prime}, G^{\prime}, G^{\prime}\right)$ where $G^{\prime}$ is considered as a right $G^{\prime}$-set by regular action.

Given $r^{\prime} \in R_{g^{\prime}}^{\prime}$ and $x^{\prime} \in X^{\prime}$, then $A=\sum_{i=1}^{n_{x^{\prime}}}\left(\alpha_{\left(x^{\prime}, i\right)}^{\prime}\right)^{x_{\left(x^{\prime}, i\right)}} \otimes \beta_{\left(x^{\prime}, i\right)}^{\prime} r^{\prime}=$

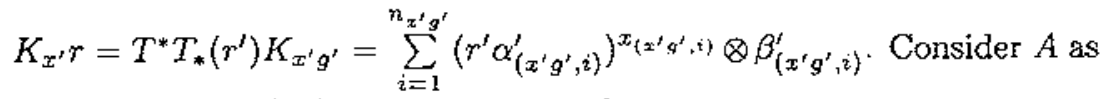
an element of $S_{*}(M)$. For every $i=1,2, \ldots, n_{x^{\prime}}$

$$
\left(r^{\prime} \alpha_{\left(x^{\prime}, i\right)}^{\prime}\right)^{x_{\left(x^{\prime}, i\right)}} \otimes \beta_{\left(x^{\prime}, i\right)}^{\prime} \in S^{*}(M)_{g^{\prime} g_{\left(x^{\prime}, i\right)} h_{\left(x^{\prime}, i\right)}^{\prime}}
$$

and

$$
\left(\alpha_{\left(x^{\prime} g^{\prime}, i\right)}^{\prime}\right)^{x_{\left(x^{\prime} g^{\prime}, i\right)} \otimes \beta_{\left(x^{\prime} g^{\prime}, i\right)}^{\prime}} r^{\prime} \in S^{*}(M)_{g_{\left(, x^{\prime}, i\right)}^{\prime} h_{\left(x^{\prime}, 2\right)}^{h^{\prime}}} g^{\prime}
$$

Therefore,

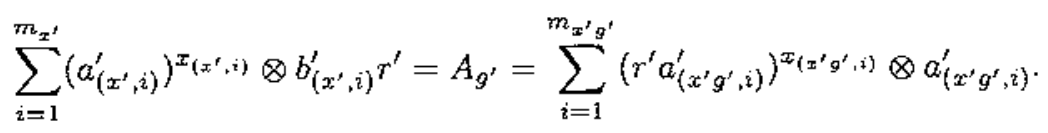

This proves (3) for $\sum$

(b) $\Rightarrow$ (a) Let

$$
\begin{aligned}
& \sum=\left\{\left(a_{\left(x^{\prime}, i\right)}^{\prime}, b_{\left(x^{\prime}, i\right)}^{\prime}, x_{\left(x^{\prime}, i\right)}\right) \in R_{g_{\left(x^{\prime}, i\right)}^{\prime}}^{\prime} \times R_{\left(g_{\left(x^{\prime}, 2\right)}^{\prime}\right)^{-1}}^{\prime} \times X \mid x^{\prime} \in X^{\prime},\right. \\
& \left.i=1,2, \ldots, n_{x^{\prime}}\right\}
\end{aligned}
$$

be a system satisfying (1), (2) and (3).

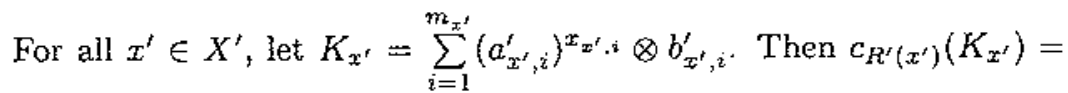
$\sum_{i=1}^{m_{x^{\prime}}} a_{x^{\prime}, i}^{\prime} b_{x^{\prime}, i}^{\prime}=1$ and for every $r^{\prime} \in R_{g^{\prime}}^{\prime}, T^{*} T_{*}\left(\lambda_{r^{\prime}}\right)\left(K_{x^{\prime} g^{\prime}}\right)=\sum_{i=1}^{m_{x^{\prime} g^{\prime}}}\left(r^{\prime} a_{x^{\prime} g^{\prime}, i}^{\prime}\right)^{x_{x^{\prime} g^{\prime}, z} \otimes}$ $a_{x^{\prime}, i}^{\prime}=\sum_{i=1}^{m_{x^{\prime}}}\left(a_{x^{\prime}, i}^{\prime}\right)^{x_{x^{\prime}, 2}} \otimes a_{x^{\prime}, i}^{\prime} r^{\prime}=K_{x^{\prime}} r^{\prime}$

By Proposition 4.2, we concluded that $T_{*}$ is separable. 
In the proof of last Proposition, we have considered $G^{\prime}$ as a right $G$ set by $g^{\prime} g=g^{\prime} \gamma(g)\left(g \in G, G^{\prime} \in G^{\prime}\right)$. Actually we can consider $G^{\prime}$ as a $G$ - $G$-set by putting $g g^{\prime}=\gamma(g) g^{\prime}$.

For every $x^{\prime} \in X^{\prime}$, the right $R$-module $T_{*}\left(R^{\prime}\left(x^{\prime}\right)\right)$ becomes an object of $g r-\left(R, G^{\prime}, G\right)$ by assigning degree $g^{\prime}$ to $r^{\prime x}$ whenever $r^{\prime} \in R_{g^{\prime}}^{\prime}$ with $x^{\prime} g^{\prime}=\xi(x)$.

We can consider $X$ as a left $G$-set by $g x=x g^{-1}(g \in G, x \in X)$. Similarly $X^{\prime}$ can be considered as a left $G^{\prime}$-set.

Moreover $\gamma(g) \xi(x)=\xi(g x)$ for every $g \in G$ and $x \in X$. Therefore, we can define two functors $T^{0}=R^{\prime} \otimes_{R}-:(G, X, R)-g r \rightarrow\left(G^{\prime}, X^{\prime}, R^{\prime}\right)-g r$ and $T_{0}:\left(G^{\prime}, X^{\prime}, R^{\prime}\right)-g r \rightarrow(G, X, R)-g r$ which are the left hand versions of $T^{*}$ and $T_{*}$. By symmetry, for every $x^{\prime} \in X^{\prime}, T_{0}\left(\left(x^{\prime}\right) R^{\prime}\right)$ is an object of $\left(G, G^{\prime}, R\right)-g r$ by assigning degree $g^{\prime}$ to $r^{\prime x}$ whenever $r^{\prime} \in R_{g^{\prime}}^{\prime}$ with $g^{\prime} x^{\prime}=\xi(x)$.

Set $S=\left(\rho, 1_{G^{\prime}}, \gamma\right)$. For every $x^{\prime} \in X^{\prime}, S^{*}\left(T_{*}\left(R^{\prime}\left(x^{\prime}\right)\right)\right)=T_{*}\left(R^{\prime}\left(x^{\prime}\right)\right) \otimes_{R}$ $R^{\prime} \in g r-\left(R^{\prime}, G^{\prime}, G^{\prime}\right)=g r-R^{\prime}$ and $S^{0}\left(T_{0}\left(\left(x^{\prime}\right) R^{\prime}\right)\right) \in\left(G^{\prime}, G^{\prime}, R^{\prime}\right)-g r=$ $R-g r$.

Lemma 4.5. For every $x^{\prime} \in X^{\prime}$ and every $g^{\prime} \in G^{\prime}, S^{*}\left(T_{*}\left(R^{\prime}\left(x^{\prime}\right)\right)\right)_{g^{\prime}}$ is isomorphic to $g^{\prime} S^{0}\left(T_{0}\left(\left(g^{\prime} x^{\prime}\right) R^{\prime}\right)\right)$.

Proof: Let $\Phi: S^{*}\left(T_{*}\left(R^{\prime}\left(x^{\prime}\right)\right)\right)_{g^{\prime}} \rightarrow g^{\prime} S^{0}\left(\left(g^{\prime} x^{\prime}\right)\left(R^{\prime}\right)\right)$ the homomorphism given by $\Phi\left(a^{\prime x} \otimes b^{\prime}\right)=a^{\prime} \otimes b^{\prime x}\left(x \in X, a^{\prime} \in R_{\sigma^{\prime}}^{\prime}, b^{\prime} \in R_{\tau^{\prime}}^{\prime}\right.$, with $x^{\prime} \sigma^{\prime}=\xi(x)$ and $\left.\sigma^{\prime} \tau^{\prime}=g^{\prime}\right)$ and extended by linearity.

$\Phi$ is well defined. Indeed, let $a^{\prime} \in R_{\sigma}^{\prime}, b^{\prime} \in R_{\tau^{\prime}, r}^{\prime} \in R_{g}$ and assume that $\sigma^{\prime} \gamma(g) \tau^{\prime}=g^{\prime}$ and $x^{\prime} \sigma^{\prime}=\xi(x)$. Then $\Phi\left(\left(a^{\prime}\right)^{x} r \otimes b^{\prime}\right)=\Phi\left(\left(a^{\prime} \rho(r)\right)^{x g} \otimes\right.$ $\left.b^{\prime}\right)=a^{\prime} \rho(r) \otimes\left(b^{\prime}\right)^{x g}=a^{\prime} \otimes r\left(b^{\prime}\right)^{g^{-1} x}=a^{\prime} \otimes\left(\rho(r) b^{\prime}\right)^{x}=\Phi\left(a^{\prime x} \otimes \rho(r) b\right)$.

And $\Phi$ is obviously an isomorphism.

Proposition 4.6. Let $T=(\rho, \xi, \gamma)$ as above. $T_{*}$ is separable if and only if $T_{0}$ is separable.

Proof: By left-right symmetry, $T_{0}$ is separable if and only if there exists a system

$$
\begin{array}{r}
\sum=\left\{\left(a_{\left(x^{\prime}, i\right)}^{\prime}, b_{\left(x^{\prime}, i\right)}^{\prime}, x_{\left(x^{\prime}, i\right)}\right) \in R_{\left(h_{\left(x^{\prime}, i\right)}^{\prime}\right)}^{\prime}\right)^{-1} \times R_{h_{\left(x^{\prime}, i\right)}^{\prime}} \times X \mid x^{\prime} \in X^{\prime}, \\
\left.i=1,2, \ldots, m_{x^{\prime}}\right\}
\end{array}
$$

satisfying the following conditions.

(I') For every $x^{\prime} \in X^{\prime}$ and $i=1,2, \ldots, m_{x^{\prime}}, h_{\left(x^{\prime}, i\right)}^{\prime} x^{\prime}=\xi\left(x_{\left(x^{\prime}, i\right)}\right)$. 
(2') For every $x^{\prime} \in X^{\prime}, \sum_{i=1}^{m_{x^{\prime}}} a_{\left(x^{\prime}, i\right)}^{\prime} b_{\left(x^{\prime}, i\right)}^{\prime}=1$.

(3) For every $r^{\prime} \in R_{h}^{\prime}$, and every $y^{\prime} \in X^{\prime}$, the following equality holds in $R^{\prime} \otimes_{R} T_{0}\left(\left(x^{\prime}\right) R^{\prime}\right)$

$$
\sum_{i=1}^{m_{h^{\prime} y^{\prime}}} a_{\left(h^{\prime} y^{\prime}, i\right)}^{\prime} \otimes\left(b_{\left(h^{\prime} y^{\prime}, i\right)}^{\prime} r^{\prime}\right)^{x_{\left(h^{\prime} y^{\prime}, i\right)}}=\sum_{i=1}^{m_{y^{\prime}}} r^{\prime} a_{\left(y^{\prime}, i\right)}^{\prime} \otimes\left(b_{\left(y^{\prime}, i\right)}^{\prime}\right)^{x_{y^{\prime}, i}}
$$

Assume that $T_{*}$ is separable. Then there exists a system

$$
\begin{aligned}
& \sum=\left\{\left(a_{\left(x^{\prime}, i\right)}^{\prime}, b_{\left(x^{\prime}, i\right)}^{\prime}, x_{\left(x^{\prime}, i\right)}\right) \in R_{g_{\left(x^{\prime}, i\right)}^{\prime}}^{\prime} \times R_{\left(g_{\left(x^{\prime}, i\right)}^{\prime}\right)^{-1}}^{\prime} \times X \mid x^{\prime} \in X^{\prime},\right. \\
& \left.i=1,2, \ldots, m_{x^{\prime}}\right\}
\end{aligned}
$$

satisfying conditions (1), (2) and (3) of Proposition 4.4. For every $x^{\prime} \in$ $X^{\prime}$ and every $i=1,2, \ldots, m_{x^{\prime}}$ let $h_{\left(x^{\prime}, i\right)}^{\prime}=\left(g_{\left(x^{\prime}, i\right)}^{\prime}\right)^{-1}$. Then

$$
\begin{array}{r}
\sum=\left\{\left(a_{\left(x^{\prime}, i\right)}^{\prime}, b_{\left(x^{\prime}, i\right)}^{\prime}, x_{\left(x^{\prime}, i\right)}\right) \in R_{\left(h_{\left(x^{\prime}, i\right)}^{\prime}\right)^{-1}}^{\prime} \times R_{h_{\left(x^{\prime}, i\right)}^{\prime}} \times X \mid x^{\prime} \in X^{\prime},\right. \\
\left.i=1,2, \ldots, m_{x^{\prime}}\right\}
\end{array}
$$

satisfies conditions ( $\left.1^{\prime}\right)$ and (2'). On the other hand, let $r^{\prime} \in R_{h^{\prime}}^{\prime}$ and $y^{\prime} \in X$. Set $g^{\prime}=h^{\prime-1}$ and $x^{\prime}=h^{\prime} y^{\prime}$. By assumption the following equality holds in $T_{*}\left(R\left(x^{\prime}\right)\right) \otimes_{R} R^{\prime}$.

$$
\sum_{i=1}^{m_{z^{\prime}}}\left(a_{\left(x^{\prime}, i\right)}^{\prime} x_{\left(x^{\prime}, a\right)} \otimes b_{\left(x^{\prime}, i\right)}^{\prime}\right) r^{\prime}=\sum_{i=1}^{m_{x^{\prime} k^{\prime}}}\left(r^{\prime} a_{\left(x^{\prime} h^{\prime}, 2\right)}^{\prime} x_{\left(x^{\prime} h^{\prime}, 2\right)} \otimes\left(b_{\left(x^{\prime} h^{\prime}, i\right)}^{\prime}\right)\right.
$$

and this element has degree $h^{\prime}$ when we view $T_{*}\left(R\left(x^{\prime}\right)\right) \otimes_{R} R^{\prime}$ as $S^{*}\left(T_{*}\left(R\left(x^{\prime}\right)\right)\right)$. Therefore, by using the isomorphism of Lemma 4.5, the following equality holds in $R^{\prime} \otimes_{R} T_{0}\left(\left(y^{\prime}\right) R^{\prime}\right)$

$$
\sum_{i=1}^{m_{h^{\prime} y^{\prime}}} a_{\left(h^{\prime} y^{\prime}, i\right)}^{\prime} \otimes\left(b_{\left(h^{\prime} y^{\prime}, i\right)}^{\prime} r^{\prime}\right)^{x_{\left(h^{\prime} y^{\prime}, 2\right)}}=\sum_{i=1}^{m_{y^{\prime}}} r^{\prime} a_{\left(y^{\prime}, i\right)}^{\prime} \otimes\left(b_{\left(y^{\prime}, i\right)}^{\prime}\right)^{x_{y^{\prime}, i}}
$$

We conclude that $T_{0}$ is separable.

Let us consider now the particular case when $R=R^{\prime}$ and $\rho$ is the identity map of $R$. By Lemma 1.8, in order to study $T^{*}$ and $T_{*}$ we can assume that $G=G^{\prime}$ and $\gamma$ is the identity map of $G$. In that case $T^{*}=T_{\xi}$ and $T_{*}=S^{\xi}$. 
Corollary 4.7. Let $R$ be a $G$-graded ring and $\xi: X \rightarrow X^{\prime}$ a morphism of right $G$-sets. The functor $T_{\xi}$ is separable.

Proof: For every $x \in X$ let $\varepsilon_{x}: S^{\xi}\left(R(\xi(x)) \rightarrow R(x)\right.$ given by $\varepsilon_{x}\left(r^{y}\right)=$ $r_{y}$ (where the $y-t h$ component of $r$ is computed in $R(x)$ ). Plainly $\varepsilon_{x}$ satisfies condition (a) of Proposition 4.3. On the other hand, if $x, y \in$ $X, g \in G, r \in R_{g}$ and $r^{\prime} \in R(\xi(x))_{\xi(y)}$, then $\varepsilon_{x}\left(\left(r r^{\prime}\right)^{y}\right)=\left(r r^{\prime}\right)_{y}=$ $\sum_{x h=y}\left(r r^{\prime}\right)_{h}=\sum_{x h=y} r\left(r^{\prime}\right)_{g^{-1} h}=r \sum_{x g h=y} r_{h}^{\prime}=r \varepsilon_{x g}\left(r^{\prime y}\right)$. Now Proposition 4.3 applies.

Corollary 4.8. Let $T=(\rho, \xi: \gamma)$ as above. If $R$ is a direct summand of $R^{\prime}$ as $R$ - $R$-bimodules, then $T^{*}$ is separable.

Proof: Consider the following commutative diagram of functors

$$
\begin{array}{cc}
g r-(R, X, G) \stackrel{T^{*}}{\longrightarrow} g r-\left(R^{\prime}, X^{\prime}, G^{\prime}\right) \\
U_{R} \downarrow & \downarrow U_{R^{\prime}} \\
\bmod -R & \stackrel{-\otimes_{R} R^{\prime}}{\longrightarrow} \\
& \bmod -R^{\prime}
\end{array}
$$

where $U_{R}$ and $U_{R^{\prime}}$ are the functor which forget the grading. Let $Y$ be a singleton and consider $G$ acting on $Y$. Let $\varphi: X \rightarrow Y$ be the only possible map. Then $\bmod -R=g r-(R, Y, G)$ and $U_{R}=T_{\varphi}$. Therefore $U_{R}$ is separable.

By Proposition 1.3 of [NVV], $-\otimes_{R} R^{\prime}$ is separable. Thus $U_{R^{\prime}} \circ T^{*}=$ $\left(-\otimes R R^{\prime}\right) \circ U_{R}$ is separable and hence $T^{*}$ is separable (see [NVV, Lemma 1.1].

Corollary 4.9. Let $R$ be a $G$-graded ring and $\xi: X \rightarrow X^{\prime}$ a morphism of right $G$-sets. The following conditions are equivolent:

(a) $S^{\xi}$ is separable.

(b) For every $x \in X$ there exists $a^{x} \in R_{e}$ such that:

(SS1) For every $x^{\prime} \in X^{\prime}$, the set $\left\{x \in \xi^{-1}\left(x^{\prime}\right) \mid a^{x} \neq 0\right\}$ is finite and

$$
\sum_{\xi(x)=x^{\prime}} a^{x}=1 .
$$

(SS2) For every $r \in R_{g}$ and $\chi \in X, a^{\chi} r=r a^{\times 9}$.

Proof: (a) $\Rightarrow$ (b) Assume that $S^{\xi}$ is separable. Let

$$
\begin{aligned}
& \sum=\left\{\left(a_{\left(x^{\prime}, i\right)}, b_{\left(x^{\prime}, i\right)}, x_{\left(x^{\prime}, i\right)}\right) \in R_{g_{\left(x^{\prime}, i\right)}} \times R_{\left(g_{\left(x^{\prime}, i\right)}\right)^{-1}} \times X \mid x^{\prime} \in X^{\prime},\right. \\
& \left.i=1,2, \ldots, m_{x}\right\}
\end{aligned}
$$


be a systcm satisfying conditions (1), (2) and (3) of Proposition 4.4(b). For any $x \in X$ let

$$
a^{x}=\sum\left\{a_{(\xi(x), i)} b_{(\xi(x), i)} \mid \dot{i}=1,2, \ldots, m_{\xi(x)}, x_{(\xi(x), i)}=x g_{\left(x^{\prime}, i\right)}\right\}
$$

If we fix an $x^{\prime} \in X^{\prime}$, then for every $x \in \xi^{-1}\left(x^{\prime}\right), a^{x}=0$, unless $x \in\left\{x_{\left(x^{\prime}, i\right)}\left(g_{\left(x^{\prime}, i\right)}\right)^{-1} \mid i=1,2, \ldots, m_{x^{\prime}}\right\}$. Therefore, $a^{x}=0$ for almost all $x \in \xi^{-1}\left(x^{\prime}\right)$.

Moreover, for a fixed $x^{\prime} \in X^{\prime}, \sum_{\xi(x)=x^{\prime}} a^{x}=\sum_{i=1}^{m_{x^{\prime}}} a_{\left(x^{\prime}, i\right)} b_{\left(x^{\prime}, i\right)}=I$. Note that this implies that $\xi$ has to be surjective.

On the other hand, if we identify $T_{*}\left(R\left(x^{\prime}\right)\right) \otimes_{R} R$ with $T_{*}\left(R\left(x^{\prime}\right)\right)$ by the canonical isomorphism, then for every $r \in R_{g}$,

$$
\sum_{i=1}^{m_{x^{\prime} g}}\left(r a_{\left(x^{\prime} g, i\right)}\right)^{x_{\left(x^{\prime} g, i\right\rangle} b_{\left(x^{\prime} g, i\right)}}=\sum_{i=1}^{m_{x^{\prime}}}\left(a_{\left(x^{\prime}, 2\right)}\right)^{x_{\left(x^{\prime}, 2\right)}} b_{\left(x^{\prime}, z\right)} r .
$$

The left hand term in the previous expression is

$$
\begin{aligned}
\mathcal{L} & =\sum_{i=1}^{m_{x^{\prime} g}}\left(r a_{\left(x^{\prime}, i\right)}\right)^{x_{\left(x^{\prime} g, 2\right)}} b_{\left(x^{\prime} g, i\right)}= \\
& =\sum_{i=1}^{m_{x^{\prime} g}}\left(r a_{\left(x^{\prime} g, i\right)} b_{\left(x^{\prime} g, i\right)}\right)^{x_{\left(x^{\prime} g, 2\right)}\left(g_{\left(x^{\prime} g, i\right)}\right)^{-1}}=\sum_{\xi(x)=x^{\prime} g}\left(r a^{x}\right)^{x} .
\end{aligned}
$$

Arguing in a similar way we obtain the following expression for the right hand term in $(*)$.

$$
\mathcal{R}=\sum_{i=1}^{m_{x^{\prime}}}\left(r_{x^{\prime}, i}\right)^{x_{x^{\prime}, 2}} s_{x^{\prime}, i} r=\sum_{\xi(x)=x^{\prime}}\left(a^{x}\right)^{x} r=\sum_{\xi(x)=x^{\prime}}\left(a^{x} r\right)^{x g} .
$$

Therefore $\left(r a^{x g}\right)^{x g}=\mathcal{L}_{x g}=\mathcal{R}_{x g}=\left(a^{x} r\right)^{x g}$ and hence $r a^{x g}=a^{x} r$ for every $x \in X$.

(b) $\Rightarrow$ (a) Let $\left\{a^{x} \mid a \in A\right\}$ be a system satisfying conditions (SS1) and (SS2) of (b). For every $x^{\prime} \in X^{\prime}$ and any $x \in \xi^{-1}\left(x^{\prime}\right)$, let $a_{\left(x^{\prime}, x\right)}=a^{x}$ and $b_{\left(x^{\prime}, x\right)}=1$. Then the system $\left\{\left(a_{\left(x^{\prime}, x\right)}, b_{\left(x^{\prime}, x\right)}, x\right) \mid x^{\prime} \in X^{\prime}, x \in\right.$ $\left.\xi^{-1}\left(x^{\prime}\right), a^{x} \neq 0\right\}$ satisfies conditions (1), (2) and (3) of Proposition 4.4(b). Indeed, conditions (1) and (2) are obvious. Let now $0 \neq r \in R_{g}$. 
Then

$$
\begin{aligned}
\sum_{x \in \xi^{-1}\left(x^{\prime}\right)}\left(a_{\left(x^{\prime}, x\right)}\right)^{x} \otimes b_{\left(x^{\prime}, x\right)} r & =\sum_{x \in \xi^{-1}\left(x^{\prime}\right)}\left(a^{x}\right)^{x} r=\sum_{x \in \xi^{-1}\left(x^{\prime}\right)}\left(a^{x} r\right)^{x g}= \\
& =\sum_{x \in \xi^{-1}\left(x^{\prime}\right)}\left(r a^{x g}\right)^{x g}=\sum_{y \in \xi^{-1}\left(x^{\prime} g\right)}\left(r a^{y}\right)^{y}= \\
& =\sum_{x \in \xi^{-1}\left(x^{\prime} g\right)}\left(r a_{\left(x^{\prime} g, x\right)}\right)^{x} \otimes b_{\left(x^{\prime} g, x\right)} .
\end{aligned}
$$

Proposition 4.4 applies to finish the proof.

Definition. Let $\xi: X \rightarrow X^{\prime}$ be a morphism of right $G$-sets. A $\xi$ separability system in a $G$-graded ring $R$ is a system $\left\{a^{x} \mid x \in X\right\}$ of elements in $R_{e}$ satisfying conditions (SS1) and (SS2) of Corollary 4.9.

Remarks. (1) Note that if $X=G, X^{\prime}$ is a singleton and $\xi: G \rightarrow X^{\prime}$ is the unique possible map, then $R$ has a $\xi$-separability system if and only if $R$ has a separability system in the sense of [Ra].

(2) Let $\xi: X \rightarrow X^{\prime}$ be a morphism of right $G$-sets and $R$ a $G$-graded ring.

(a) If $S_{\xi}$ is separable (or cquivalently, if $R$ has a $\xi$-separability systcm), then $\xi$ is epic.

(b) If $\xi$ is epic and for every $x^{\prime} \in X^{\prime}, \xi^{-1}\left(x^{\prime}\right)$ is finite with order invertible in $R$, then $a^{x}=\left|\xi^{-1}(\xi(x))\right|^{-1}$ is a $\xi$-separability system whenever $\left|\xi^{-1}\left\langle x^{\prime}\right)\right|$ is constant in each orbit of $X^{\prime}$.

(c) Assume that $\xi$ is epic. For every orbit $Y$ in $X$ let $\xi_{Y}: Y \rightarrow \xi(Y)$ be the restriction of $\xi$ to $Y$. $R$ has a $\xi$-separability system if and only if it has a $\xi_{Y}$-separability system for every orbit $Y$ of $X$. Namely, if for every orbit $Y$ in $X,\left\{a^{y} \mid y \in Y\right\}$ is a $\xi_{Y}$-separability system for $R$, then $\left\{a^{x} \mid x \in X\right\}$ is a $\xi$-separability system for $R$.

\section{Weak dimension}

Let $G$ be a group, $H$ a subgroup of $G$ and $R$ a $G$-graded ring.

Let $G / H$ be the set of left $H$-cosets and $\varphi_{H}: G \rightarrow G / H$ the canonical projection. In order to simplify the notation let us denote by $T_{H}$ and $S_{H}$ the functors $T_{\varphi_{H}}: R-g r \rightarrow(R, G / H)-g r$ and $S^{\varphi_{H}}:(G / H, R)-g r \rightarrow$ $R-g r$ respectively.

For a (graded) ring ( $g r). w \cdot \operatorname{dim}(R)$ will denote the (graded) weak dimension of $R$. Similarly, for a $R$-module, $f . d .(M)$ will denote the flat dimension of $M$. Recall that, for a graded module ${ }_{R} M$, the graded flat dimension coincides with the flat dimension (see e.g. [NV1]).

Next theorem extends Theorem 7 in [Rí1] and gives an approach to answer Question 2 in the samc paper. 
Theorem 5.1. Let $R$ be a $G$-graded ring. Assume that for every finitely generated subgroup $H$ of $G$ the functor $S_{H}$ is separable. Then $g r . w \cdot \operatorname{dim}(R)=w \cdot \operatorname{dim}(R)$.

Remark. Note that if $S_{H}$ is separable, then the $H$-graded ring $R_{(H)}=$ $\underset{g h \in H}{\oplus} R_{h}$ has a separability system (in the sense of [Ra]). Indeed, $S_{H}$ is separable if and only if $R$ has a $\varphi_{H}$-separability system. But if $\left\{x^{g} \mid g \in\right.$ $G\}$ is a $\varphi_{H}$-separability system, then $\left\{x^{h} \mid h \in H\right\}$ is a separability system for $R_{(H)}$.

The converse is not true in general as the following example shows.

Example. Let $K$ be a field of characteristic 2. Let $G=\mathrm{Z}_{2} \times \mathrm{Z}_{2}$ and $H$ the subgroup of $G$ generated by $(1,1)$. Consider the ring $R$ of the matrices of the form $\left(\begin{array}{lll}a & b & c \\ 0 & a & 0 \\ 0 & 0 & a\end{array}\right)$ with entries in $K . R$ has a $G$-grading given by:

$$
\begin{array}{lll}
R_{(0,0)}=\left\{\left(\begin{array}{ccc}
a & 0 & 0 \\
0 & a & 0 \\
0 & 0 & a
\end{array}\right) \mid a \in K\right\}, & R_{(1,1)}=0 \\
R_{(1,0)}=\left(\begin{array}{ccc}
0 & K & 0 \\
0 & 0 & 0 \\
0 & 0 & 0
\end{array}\right), & R_{(0,1)}=\left(\begin{array}{ccc}
0 & 0 & K \\
0 & 0 & 0 \\
0 & 0 & 0
\end{array}\right) .
\end{array}
$$

Then $\left\{a^{(0,0)}=\left(\begin{array}{lll}1 & 0 & 0 \\ 0 & 1 & 0 \\ 0 & 0 & 1\end{array}\right), a^{(1,1)}=0\right\}$ is a separability system for $R_{(H)}$. But $R$ has not a $\varphi_{H}$-scparability system. Indeed, if $\left\{a^{g} \mid g \in G\right\}$ is a $\varphi_{H}$-Separability system, then $a^{(1,0)}\left(\begin{array}{lll}0 & 1 & 0 \\ 0 & 0 & 0 \\ 0 & 0 & 0\end{array}\right)=\left(\begin{array}{lll}0 & 1 & 0 \\ 0 & 0 & 0 \\ 0 & 0 & 0\end{array}\right) a^{(0,0)}$ and $a^{(0,1)}\left(\begin{array}{lll}0 & 0 & 1 \\ 0 & 0 & 0 \\ 0 & 0 & 0\end{array}\right)=\left(\begin{array}{lll}0 & 0 & 1 \\ 0 & 0 & 0 \\ 0 & 0 & 0\end{array}\right) a^{(0,0)}$. Therefore $a^{(1,0)}=a^{(0,0)}=$ $a^{(0,1)}$ and $1=a^{(0,1)}+a^{(1,0)}=2 a^{(1,0)}=0$.

Lemma 5.2. Let ${ }_{R} P$ be a projective left $R$-module and $M \in$ $\operatorname{Gen}\left({ }_{R} P\right)$. Then ${ }_{R} M$ is projective if and only if it is projective in the cotegory $\operatorname{Gen}\left({ }_{R} P\right)$.

Proof: Let $f: K \rightarrow L$ be a homomorphism in the category $\operatorname{Gen}\left({ }_{R} P\right)$. If $f$ is surjective, then $f$ is an epimorphism in the category $\operatorname{Gen}\left({ }_{R} P\right)$. 
Conversely, if $f$ is an epimorphism in the category $\operatorname{Gen}\left({ }_{R} P\right)$ and $C$ is the cokernel of $f$, then the canonical projection $p: L \rightarrow C$ is also a homomorphism in $\operatorname{Gen}\left({ }_{R} P\right)$ such that $p$ of $=0$. Thus $C=0$, this implies that $f$ is surjective.

Since ${ }_{R} M \in \operatorname{Gen}\left({ }_{R} P\right)$, then there exists an epimorphism $f: P^{(I)} \rightarrow M$ which is an epimorphism both in $\operatorname{Gen}\left({ }_{R} P\right)$ and $R$-mod. Thus ${ }_{R} M$ is simultaneously projective in $R-\bmod$ and $\operatorname{Gen}\left({ }_{R} P\right)$.

Let $\mathcal{C}$ be a Grothendieck category. Recall that an object $F$ in $C$ is said to be flat if for every finitely presented object $M$ in $C$ and every epimorphism $f: N \rightarrow F$, the correspondent homomorphism of abelian groups $\operatorname{Hom}_{C}(M, f): \operatorname{Hom}_{\mathcal{C}}(M, N) \rightarrow \operatorname{Hom}_{\mathcal{D}}(M, F)$ is an epimorphism (see e.g. [Po]).

When $I$ is an idempotent left ideal of a ring $R$, then $\operatorname{Gen}\left({ }_{R} I\right)$ is a Grothendieck category (see e.g. [W]) because it is closed under submodules. Of course, a left ideal with local units is an idempotent idcal.

Proposition 5.3. Let $I$ be a left ideal of $R$ with local units. Then, an object $F$ in $\operatorname{Gen}\left({ }_{R} I\right)$ is flat in this category if and only if it is flat os a left $R$-module.

Proof: Since $\operatorname{Gen}\left({ }_{R} I\right)$ is closed under subobjects, the finitcly generated (resp. finitely presented) objects of $\operatorname{Gen}\left({ }_{R} I\right)$ are just the finitely generated (resp. finitely presented) left $R$-modules generated by ${ }_{R} I$. Moreover, as we have remarked in the proof of the previous lemma the epimorphisms in $\operatorname{Gen}\left({ }_{R} I\right)$ are the epimorphisms in $R$-mod between objects in $\operatorname{Gen}\left({ }_{R} I\right)$. Therefore the necessary condition is obvious.

Let now $F$ be a flat object in $\operatorname{Gen}\left({ }_{R} I\right)$. First we will sce that for every idempotent $e \in I, e F$ is flat as a left $e R e$-module. Consider $R e$ as an $R-e R e$-bimodule and the functors $H=\operatorname{Hom}\left({ }_{R} R e,-\right): R$-mod $\rightarrow$ $e R e-\bmod$ and $T=e R \otimes_{e R e}-: e R e-\bmod \rightarrow R-\operatorname{Mod}$. Let $\mu: 1_{e R e-\bmod } \rightarrow$ $H T$ and $\varepsilon: T H \rightarrow 1_{R-\text { mod }}$ be the unit and the counit of the adjoint pair $(T, H)$.

Let $M$ be a finitely presented left $e R e$-module and $f: M \rightarrow e F$ a homomorphism of left $e$ Re-modules, then $T(M)$ is a finitely presented left $R$-module in $\operatorname{Gen}(R I)$. Let $p: I^{(X)} \rightarrow F$ be an epimorphism. By flatness of $F$ in $\operatorname{Gen}\left({ }_{R} I\right)$ there exists $g: T(M) \rightarrow I^{(X)}$ such that $p \circ g=$ $\varepsilon_{F} \circ T(f)$. Since $R e \otimes_{e R e} M$ is finitely generated, there exists $Y \subseteq X$ finite such that $\operatorname{Im}(g) \subseteq I^{(Y)}$ and hence therc exist $g^{\prime}: T(M) \rightarrow I^{(Y)}$ and $p^{\prime}: I^{(Y)} \rightarrow F$ such that $p^{\prime} \circ f^{\prime}=\varepsilon_{F} \circ T(f)$. Applying the functor 
$H$, one has a commutative diagram

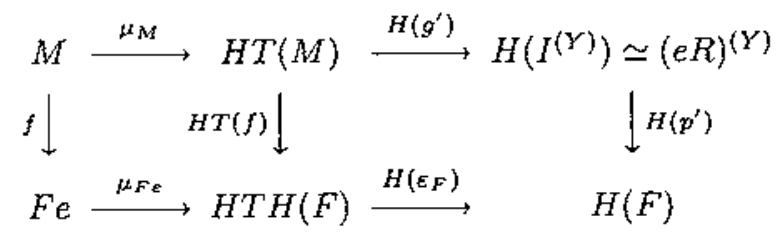

but $\mu_{X}$ is an isomorphism for every $X \in e$ Re-mod. On the other hand $H\left(\varepsilon_{F}\right) \circ \mu_{H(F)}=1_{H(F)}$. Thus $f$ factors throught ${ }_{e R e}(R e)^{(Y)}$ which is projective and finitely generated and hence $f$ factors throughout a finite product of copies of $e$ Re. This proof that $e R e e F$ is flat.

Now we prove that ${ }_{R} F$ is flat. Let $J$ be a right ideal of $R$ and assume that $\sum_{i=1}^{n} r_{1} m_{1}=0$ for $m_{1}, m_{2}, \ldots, m_{n} \in F$ and $r_{1}, r_{2}, \ldots, r_{n} \in J$. Let $e$ be an idempotent in $I$ such that $e m_{1}=m_{1}$ for every $i=1,2, \ldots, n$. Then $\sum_{i=1}^{n} r_{1} \otimes m_{1}=\sum_{i=1}^{n} r_{1} \otimes e m_{1}=\sum_{i=1}^{n} r_{1} e \otimes e m_{1}$. Therefore $\sum_{i=1}^{n} r_{1} e \otimes e m_{1}$ belongs to the kernel of the canonical $J e \otimes_{e R e} e F \rightarrow e R e$, which is injective. Therefore it is 0 in $J e \otimes_{\mathrm{e} R e} e F$ and hence it is 0 in $J \otimes_{R} F$.

Lemma 5.4. Let $\xi: X \rightarrow X^{\prime}$ be a morphism of left $G$-sets and assume that $G$ acts freely on $X$. For every $x^{\prime} \in X^{\prime}, S_{\xi}\left(\left(x^{\prime}\right) R\right)$ is isomorphic to $\underset{\xi(x)=x^{\prime}}{\oplus}(x) R$. Therefore $S_{\xi}$ preserves projectivity.

Proof: For every $x \in \xi^{-1}\left(x^{\prime}\right)$ set $f_{x}:(x) R \rightarrow S^{\xi}\left(\left(x^{\prime}\right) R\right)$ given by: $f_{x}(r)=r^{y}$ if $r \in{ }_{y}(x) R$. Note that if $r \in R_{g}$ and $g x=y$ then $g x^{\prime}=$ $\xi(y)$ and hence $r \in \xi(y)\left(x^{\prime}\right) R$. If $r \in R_{g}$ and $s \in R_{h}$, then $f_{x}(r s)=$ $(r s)^{g h x}=r(s)^{h x}=r f_{x}(s)$. Thus $f_{x}$ is a homomorphism of left $R$ modules and it is obviously graded. Therefore there is an homomorphism $f: \underset{\xi(x)=x^{\prime}}{\oplus}(x) R \rightarrow S_{\xi}\left(\left(x^{\prime}\right) R\right)$ such that $f$ restricted to $(x) R$ is equal to $f_{x}$ for every $x \in \xi^{-1}\left(x^{\prime}\right)$.

Now we show that $f$ is bijective. Let $\sum_{x \in X} r^{x} \in \operatorname{Ker}(f)$ where $r^{x} \in(x) R$. Since $f$ is a graded homomorphism, then $\sum_{x \in X} y^{y}\left(r^{x}\right) \in \operatorname{Ker}(f)$ for any $y \in X$. If $g \in \operatorname{Supp}\left({ }_{y}\left(r^{x}\right)\right) \cap \operatorname{Supp}\left({ }_{y}\left(r^{x^{\prime}}\right)\right)$, then $g x=y=g x^{\prime}$, therefore $x=x^{\prime}$. Thus $0=f\left(\sum_{x \in X} y\left(r^{x}\right)\right)=\left(\sum_{x \in X} y\left(r^{x}\right)\right)^{y}$ and hence ${ }_{y}\left(r^{x}\right)=0$ for every $x, y \in X$. This proof that $f$ is injective. On the other hand, if $r^{y} \in S_{\xi}\left(\left(x^{\prime}\right) R\right)_{y}$, then $r \in \xi(y)\left(x^{\prime}\right) R$. Therefore, $g x^{\prime}=\xi(y)$ for every $g \in \operatorname{Supp}(r)$. For every $g \in G$, let $x_{g}=g^{-1} y$. Then $r_{g} \in{ }_{y}\left(x_{g}\right) R$ and $f_{x_{g}}\left(r_{g}\right)=\left(r_{g}\right)^{y}$. thus $f\left(\sum_{g \in G} r_{g}^{x_{g}}\right)=r^{y}$. 
Now if $P \in\left(G, X^{\prime}, R\right)-g r$ is projective, then it is a direct summand of copies of modules of the form $\left(x^{\prime}\right) R$ for some $x^{\prime} \in X^{\prime}$. Since $S^{\xi}$ preserves direct sums, $S^{\xi}(P)$ is isomorphic to a direct summand of a direct sum of modules of the form $(x) R$ for some $x \in X$. Therefore $S^{\xi}(P)$ is projective.

Let $R$ be a $G$-graded ring. Let $P=\sum_{g \in G} R(g)$. For every $g \in G$ let

$$
u_{g}: R(g) \longrightarrow P
$$

the canonical monomorphism. Consider $P$ as an $G \times G$-graded $R-R$ bimodule as in Section 2. For every subgroup $H$ of $G$, we will consider $G$ as an $G-H$-set. We will use the following notation:

$$
\begin{aligned}
& A_{H}=\operatorname{END}\left(P_{R}\right)_{H} \\
& J_{H}=\left\{\alpha \in A_{H} \mid \alpha u_{x}=0 \text { for almost all } g \in G\right\} \\
& U_{H}=\underset{C \in G / H}{\oplus} R(C) \text { considered as an } G / H \times G / H \text {-graded bimodule as }
\end{aligned}
$$
in Section 2.

$S_{H}=\operatorname{End}_{g r-(R, G / H)}(U)$

$I_{H}=\left\{\alpha \in S_{H} \mid \alpha u_{C}=0\right.$ for almost all $\left.C \in G / H\right\}$.

Lemma 5.5. $J_{H}$ is projective as a left $A_{H}$-module.

Proof: Actually $J_{H}=\underset{g \in G}{\oplus} A_{H} P_{g}$.

Proof of Theorem 5.1:

First it is well known that $g r . w \cdot \operatorname{dim}(R) \leq w \cdot \operatorname{dim}(R)$ (see c.g. [NV1]).

Assume that $g r . w . \operatorname{dim}(R) \leq n$. Let $H$ be a finitely generated subgroup of $G$ and $M \in(G / H, R)-g r$. Let

$$
\cdots \longrightarrow P_{2} \stackrel{f_{2}}{\longrightarrow} P_{1} \stackrel{f_{1}}{\longrightarrow} P_{0} \stackrel{f_{0}}{\longrightarrow} M \longrightarrow 0
$$

be a projective resolution in $(G / H, R)-g r$. Then by Lemma 5.4

$$
\cdots \longrightarrow S_{H}\left(P_{2}\right) \stackrel{S_{H}\left(f_{2}\right)}{\longrightarrow} P_{l} \stackrel{S_{H}\left(f_{1}\right)}{\longrightarrow} P_{0} \stackrel{S_{H}\left(f_{0}\right)}{\longrightarrow} M \longrightarrow 0
$$

is a projective resolution in $R-g r$. Therefore, $\operatorname{Ker}\left(S_{H}\left(f_{n-1}\right)\right)$ is flat (being $\left.f_{-1}=0: M \rightarrow 0\right)$. But $\operatorname{Ker}\left(S_{H}\left(f_{n-1}\right)\right)=S_{H}\left(\operatorname{Ker}\left(f_{n-1}\right)\right)$ and $S_{H}$ is separable, therefore $\operatorname{Ker}\left(f_{n-1}\right)$ is flat. Thus for every $M \in$ $(G / H, R)-g r, f d(M) \leq n$.

Let $B=\overline{\operatorname{END}}\left({ }_{R} P\right)_{H}$. Then $J_{H}=\left\{\alpha \in B \mid u_{g} \alpha=0\right.$ for almost all $g \in G]\}$. By Theorem $3.4(G / H, R)-g r$ is equivalent to $\operatorname{Gen}\left(s_{H}\left(I_{H}\right)\right)$. 
By Corollary $3.10, \operatorname{Gen}\left({ }_{B}\left(J_{H}\right)\right)$ is equivalent to $\operatorname{Gen}\left(s_{H}\left(I_{H}\right)\right)$. Finally, by Lemma $3.5 \mathrm{Gen}\left(B_{B}\left(J_{H}\right)\right)$ is equivalent to $\operatorname{Gen}\left(A_{H}\left(J_{H}\right)\right)$. Thus $f d(M) \leq n$ for all $M \in \operatorname{Gen}\left(A_{H}\left(J_{H}\right)\right)$.

For every subgroup $H$ of $G, I_{G}$ is isomorphic to $\left(I_{H}\right)^{(G / H)}$ as a right $A_{H}$-module. Thus $\operatorname{Gen}\left(A_{H}\left(I_{G}\right)_{A_{H}}\right)=\operatorname{Gen}\left(A_{H}\left(I_{H}\right)\right)$.

Let $M \in \operatorname{Gen}\left(A_{G}\left(J_{G}\right)\right)$ and

$$
\cdots \rightarrow P_{2} \stackrel{f_{2}}{\longrightarrow} P_{1} \stackrel{f_{1}}{\longrightarrow} P_{0} \stackrel{f_{0}}{\longrightarrow} M \longrightarrow 0
$$

a projective resolution of $M$ such that $P_{n} \in \operatorname{Gen}\left(A_{G}\left(J_{G}\right)\right)$ for cvery $n \geq$ 0 . Note that such a projective resolution exists because $\operatorname{Gen}\left(A_{G}\left(J_{G}\right)\right)$ is closed under submodules and ${ }_{A_{G}}\left(J_{G}\right)$ is projective (see Lemma 5.5). Since $A_{H}\left(A_{G}\right)$ is projective and restriction of scalars sends modules in $\operatorname{Gen}\left(A_{G}\left(J_{G}\right)\right)$ to modules in $\operatorname{Gen}\left(A_{H}\left(J_{G}\right)\right)$, then $A_{H} \operatorname{Ker}\left(f_{n-1}\right)$ is flat. But $A_{G}$ is the direct limit of the $A_{H}$ 's where $H$ runs on the finitely generated subgroups of $G$. Therefore $A_{H} \operatorname{Ker}\left(f_{n-1}\right)$ is flat and hence $f d\left(A_{G} M\right) \leq n$ for every $M \in \operatorname{Gen}\left(A_{G}\left(J_{G}\right)\right)$.

Now by using the equivalence $\operatorname{Gen}\left(A_{G}\left(J_{G}\right)_{A_{G}}\right) \cong \operatorname{Gen}\left(B_{G} J\right)$ of Lemma 3.5 one has that $f d\left(B_{G} M\right) \leq n$ for every $M \in \operatorname{Gen}\left(B_{G}\left(J_{G}\right)\right)$. And using the equivalence $\operatorname{Gen}\left(B_{G}\left(J_{G}\right)\right) \cong \operatorname{Gen}\left(s_{G} I_{G}\right)=R-\bmod$ of Corollary 3.10 for $G=X=H$ one has that $w \cdot \operatorname{dim}(R) \leq n$.

\section{References}

[A] T. ALBU, Pure ideals, quotient categories and infinite groupgraded rings, Comm. in Algebra 18(3) (1990), 839-862.

[AN] T. ALBU AND C. NĂSTĂSESCU, Infinite group-graded rings, rings of endomorphisms and localization, J. of Pure and Appl. Algebra 59 (1989), 125-150.

[AM] P. N. ANH AND L. MÁRKI, Morita equivalence for rings without identity, Tsukuba J. Math. 11 (1987), 1-16.

[AW] P. N. ANH AND R. WIEGANDT, On Grothendieck categories: Morita duality, Preprint.

[B1] M. BEATTIE, A Generalization of the smash product of a graded ring, J. of Pure and Appl. Algebra 52 (1988), 219-226.

[B2] M. BEATTIE, Duality theorems for rings with actions and coactions, J. Algebra 115 (1988), 302-312.

[CM] M. COHEN ANo S. MONTGOMERY, Group graded rings, smash product and group actions, Trans. Am. Math. Soc. 284 (1984), $237-258$. 
[CF] R. R. COLBy AND K. R. Fuller, Exactness of the double dual and Morita duality for Grothendieck categories, J. Algebra $\mathbf{8 2}$ (1983), 546-558.

[D1] E. C. DADE, Clifford Theory for group graded rings, J. Reine Angew. Math. 369 (1986), 40-86.

[D2] E. C. DADE, Clifford Theory for group graded rings II, J. Reine Angew. Math. 387 (1988), I48-181.

[F] K. R. Fuller, Density and equivalences; $J$. of Algebra 29 (1974), 528-550.

[GG] J. L. Gómez Pardo and P. A. Guil Asensio, Linear compactness and Morita duality for Grothendieck categories, to appear in J. Algebro.

[GN] J. L. GÓMEZ PARDO AND C. NĂSTĂSFSCU, Relative projectivity, graded Clifford theory and applications, J. Algebra 141 (1991), 484-504.

[M] C. Menini, Functors between categories of graded modules. Applications, Preprint.

[MN] C. MENINI AND C. NĂSTĂSESCU, When are induced and coinduced functors equivalent?, Preprint.

[MR] C. MENINI AND A. DEI, Río, Morita duality and graded rings, Comm. in Algebro 19 (1991), 1765-1794.

[NRV] C. Năstăsescu, S. RAianu and F. VAN OYstaeyen, Modules graded by $G$-sets, Math. Z. 203 (1990), 605 627.

[NVV] C. NĂSTĂSESCl; M. VAN DER BERGH AND F. VAN OYSTAEYF, Separable functor, applications to graded rings and modules, J. Algebra 123 (1989), 397-413.

[NV1] C. NĂstăsESCl AND F. VAN OYSTAEYEN, "Graded ring theory," North Holland Mathematical Library 28 (North Holland, Amsterdam, 1982).

[NV2] C. NĂSTĂSESCU AND F. VAN OYSTAEYEN, Clifford Theory for subgroups of graded groups, Preprint.

[Po] N, POPESCU, "Abelion categories with applications to rings and modules," LMS Monographs 3, Acad. Press, 1973.

[Q] D. Quin,, Group-graded rings and duality, Trans. A.M.S. 292.

[Ra] M. D. RAFAEL, Separable functors revisited, Comm. in Algebra 18 (1990), 1445-1459.

[Ri1] A. DEL Rio, Weak dimension of group-graded rings, Publicacions Matemàtiques 34 (1990), 209-216.

[Rí2] A. DEL Río, Graded rings and equivalences of categories, Comm. in Algebra 19 (1991), 997-1012. 
[S] B. STENSTRÖM, "Ring of quotients," Springer-Verlag, New YorkHeidelberg-Berlin, 1975.

[W] R. WISBAUER, Localization of modules and the central closure of rings, Comm. in Algebra 9 (1981), 1455-1493.

Departamento de Matemáticas

Universidad de Murcia

30071 Murcia

SPAIN

Rebut el 7 de Gener de 1992 